\title{
eNeuro
}

Research Article: Theory/New Concepts / Integrative Systems

\section{Linking brain structure, activity and cognitive function through computation}

https://doi.org/10.1523/ENEURO.0316-21.2022

Cite as: eNeuro 2022; 10.1523/ENEURO.0316-21.2022

Received: 26 July 2021

Revised: 11 January 2022

Accepted: 17 January 2022

This Early Release article has been peer-reviewed and accepted, but has not been through the composition and copyediting processes. The final version may differ slightly in style or formatting and will contain links to any extended data.

Alerts: Sign up at www.eneuro.org/alerts to receive customized email alerts when the fully formatted version of this article is published.

Copyright (C) 2022 Amunts et al.

This is an open-access article distributed under the terms of the Creative Commons Attribution 4.0 International license, which permits unrestricted use, distribution and reproduction in any medium provided that the original work is properly attributed. 


\section{Linking brain structure, activity and cognitive function through computation}

3

4

\section{Abbreviated title}

\section{Computing the brain}

\section{List of all authors and affiliations}

Katrin Amunts

${ }^{1}$ Institute of Neurosciences and Medicine (INM-1), Research Centre Jülich, 52425 Jülich, LeoBrandt-Strasse, Germany

${ }^{2}$ C. \& O. Vogt Institute for Brain Research, University Hospital Düsseldorf, Heinrich-Heine University Düsseldorf, Merowingerplatz 1a, 40225 Düsseldorf, Germany Javier DeFelipe

${ }^{3}$ Laboratorio Cajal de Circuitos Corticales, Centro de Tecnología Biomédica, Universidad Politécnica de Madrid. Pozuelo de Alarcón, 28223 Madrid, Spain

${ }^{4}$ Instituto Cajal, Consejo Superior de Investigaciones Científicas (CSIC), Avda. Doctor Arce 37, 28002 Madrid, Spain

Cyriel Pennartz

${ }^{5}$ Cognitive and Systems Neuroscience Group, Swammerdam Institute for Life Sciences, University of Amsterdam, Amsterdam, Netherlands, Sciencepark 904, 1098 XH, Amsterdam.

Alain Destexhe

${ }^{6}$ Paris-Saclay University, CNRS, Institute of Neuroscience (NeuroPSI), Gif sur Yvette, France Michele Migliore

${ }^{7}$ Institute of Biophysics, National Research Council, via Ugo La Malfa 153, 90146

Palermo, Italy

Philippe Ryvlin

${ }^{8}$ Department of Clinical Neurosciences, CHUV, Rue du Bugnon 46, CH-1011, Lausanne,

Switzerland

Steve Furber

${ }^{9}$ Department of Computer Science, The University of Manchester, Oxford Road, Manchester M13

9PL, UK

Alois Knoll

${ }^{10}$ Department of Informatics, Technical University of Munich, Boltzmannstr. 3

85748 Garching, Germany

Lise Bitsch

${ }^{11}$ The Danish Board of Technology Foundation, Copenhagen, Denmark Jan G. Bjaalie

${ }^{12}$ Institute of Basic Medical Sciences, University of Oslo, Oslo, Norway

Yannis loannidis

${ }^{13}$ ATHENA Research \& Innovation Center, Greece

Thomas Lippert

${ }^{14}$ Institute for Advanced Simulation (IAS), Jülich Supercomputing Centre (JSC), Research Centre Jülich, Jülich, 52425 Jülich, Leo-Brandt-Strasse, Germany

Maria V. Sanchez-Vives

${ }^{15}$ ICREA and Systems Neuroscience, Institute of Biomedical Investigations August Pi i Sunyer, 08036, Barcelona, Spain

Rainer Goebel

${ }^{16}$ Department of Cognitive Neuroscience, Department of Cognitive Neuroscience, Faculty of Psychology and Neuroscience, Maastricht University, Oxfordlaan 55, 6229 EV Maastricht, The Netherlands

Viktor Jirsa

${ }^{17}$ Aix Marseille Université, Institut National de la Santé et de la Recherche Médicale, Institut de 


\section{Author contributions}

Katrin Amunts designed and performed research, designed and wrote the paper Javier DeFelipe designed and performed research, wrote the paper Cyriel Pennartz designed and performed research, wrote the paper Alain Destexhe designed and performed research, wrote the paper Michele Migliore designed and performed research, wrote the paper Philippe Ryvlin designed and performed research, wrote the paper Steve Furber designed and performed research, wrote the paper Alois Knoll designed and performed research, wrote the paper Lise Bitsch designed and performed research, wrote the paper Jan G. Bjaalie designed and performed research, wrote the paper Yannis loannidis designed and performed research, wrote the paper Thomas Lippert designed and performed research, wrote the paper Maria V. Sanchez-Vives designed and performed research, wrote the paper Rainer Goebel designed and performed research, wrote the paper Viktor Jirsa designed and performed research, designed and wrote the paper

5. Correspondence should be addressed to:

Katrin Amunts; Institute of Neurosciences and Medicine (INM-1), Research Centre Jülich, 52425 Jülich, Germany

Email address: k.amunts@fz-juelich.de

6. Number of figures: 5 figures, 4 boxes

7. Number of tables : non

8. Number of multimedia: non

9. Number of words for Abstract: 174

10. Number of words for Significance statement: 120

11. Number of words for Introduction: 396

12. Number of words for Conclusion: 481

13. Acknowledgements: The Human Brain Project is a collaborative, interdisciplinary effort including groups from more than 20 countries. Without the enduring engagement, scientific curiosity and hard work of the entire HBP consortium, and the support of their research institutions, the presented work would not have been possible.

14. Conflict of interest: All authors are leading Pls in the Human Brain Project, a European Flagship project (https://www.humanbrainproject.eu/en/ ). As such, they are responsible for the development of the digital research infrastructure EBRAINS.

15. Funding sources: This project has received funding from the European Union's Horizon 2020 Framework Programme for Research and Innovation under the Specific Grant Agreement No. 945539 (Human Brain Project SGA3). 
Abstract

Understanding the human brain is a 'Grand Challenge' for 21st century research. Computational approaches enable large and complex datasets to be addressed efficiently, supported by artificial neural networks, modeling and simulation. Dynamic generative multiscale models, which enable causation across scales and are guided by principles and theories of brain function, are instrumental to link brain structure and function. This integrated approach to neuroscientific discovery is framed within the BigBrain, which spatially anchors tissue models and data across different spatial scales and assures that multiscale models are supported by the data, making the bridge to both basic neuroscience and medicine. Research at the cross-over of neuroscience, computing and robotics has the potential to push neuro-inspired technologies, taking advantage of a growing body of insights into perception, plasticity and learning. To render data, tools and methods, theories, basic principles and concepts interoperable, the Human Brain Project has launched EBRAINS, a digital neuroscience research infrastructure, building a transdisciplinary community of researchers united by the quest to understand the brain, with fascinating insights and perspectives for societal benefits.

\section{Significance statement}

Theoretical and methodological integration leads to consolidation and deeper intuitive understanding, without which scientific progress remains unguided. In 2013 the European Union launched the Human Brain Project (HBP) with the mission to integrate spatial and temporal scales of brain sciences within a common framework, ultimately leading to the digital research infrastructure EBRAINS. It has become evident that doing science in EBRAINS will require a culture change, unknown to the neuroscientific community albeit common in other large-scale projects such as elementary particle physics. The novel HBP-style neuroscience is characterized by transparent domain boundaries and deep integration of highly heterogeneous data, models, and information technologies. In this article HBP scientists exemplify their science case and illustrate the capacity of the EBRAINS ecosystem. 
Advances in science have been driven by the human search for knowledge and understanding of nature, from the world around us to principles governing the whole universe. But there is a universe inside each one of us that manifests and defines our consciousness, cognition, behavior, emotions, health and illness, a universe that remains relatively unexplored yet contains the secrets of our human nature. It gives rise to behavior that we are all familiar with, allowing us to communicate, but also to manipulate information, be creative and spontaneous, make informed decisions, reason about moral and ethical questions and much more. Human curiosity has driven researchers forward to search for knowledge and understanding of this universe, which is per se a legitimate human endeavor. This search, however, is most challenging due to the complexity of the brain. Similar to research into other complex systems, brain research benefits from computational analysis tools as well as from new forms of collaboration, including large national and international consortia. Compared to other research disciplines such as nuclear physics or astronomy, such largescale collaboration is not so common in the fields of neuroscience and medicine. It is, however, not by chance that large national and international projects devoted to brain investigation have surfaced around the world in the last decade (Adams et al., 2020; Quaglio et al., 2021).

The present paper will:

- $\quad$ Provide a brief overview of the present status of key aspects of brain research and related challenges towards a deeper understanding of brain complexity

- Motivate research focused on the multi-level organization of the brain, both in space and time, and to better understand the rules by which observations at a lower scale influence those at the higher one, and vice versa

- Highlight the role of theory, brain modelling and simulation to explore the multiscale organization of the brain

- $\quad$ Argue for the need to develop new tools for data analytics, brain-inspired learning, neurorobotics and atlasing of the brain under a common roof, i.e., a joint research infrastructure

- $\quad$ Elucidate how the European Human Brain Project (HBP) is contributing to brain research and why it is developing EBRAINS as a new research infrastructure, in a co-design approach between neuroscientists and developers, engineers and informaticists

- Indicate the perspectives for brain medicine arising therefrom

- Illustrate the potential for the development of brain-inspired computing, technology and high-performance computing

163 - Emphasize collaborative approaches

- $\quad$ Provide conclusions for future research

\section{Brain complexity}

The human brain is organized across different spatial scales - from molecules in the Angström and nanometer range, to cells on micrometer scales, local neuronal circuits, to whole brain networks at the centimeter scale, and functional systems underlying, for example, cognition and consciousness. As each level is unique in its organization of constituents and their activities, first principles nevertheless exist and account of functional or computational architectures that hold at multiple scales. Examples of this are the free energy principle and 'synergetics' that explain self-organization and pattern 
formation at multiple scales (Friston et al., 2015; Friston et al., 2017; Haken, 1983; Huys et al., 2014; Kiebel and Friston, 2011). The principles provide guidance realizing the computational processes and optimizing neuroanatomical and neurochemical structures, and the data provide the building blocks for the microcircuitry and networks across spatial and temporal dimensions. For instance, molecules may change their conformation within a few milliseconds, while other processes occur during the whole lifespan, over many decades.

Thus, functional architectures in the brain can be conceptualized at different scales of spatiotemporal organization, wherein molecular and cellular processes are subsumed under macroscopic functional entities like multi-area brain systems influencing behavior. Nerve cells are key components within this multi-level organization, and are themselves intricate autonomous structures - with a nucleus hosting genetic information, organelles involved in the production of proteins and metabolism, bilipid membranes in which receptors and other molecules are embedded, and trees of axons and dendrites with spines. The activities of most of these constituents, if not all, are organized in networks establishing a set of causal interactions, the Interactome (Klein et al., 2021). Distinct anatomical networks display a hierarchical architecture with multiple nodes of convergence of afferents and divergence of efferents, providing the substrate for both serial and parallel processing. Furthermore, neuronal circuit activity with excitatory and inhibitory mechanisms of signal transduction is highly influenced by neuromodulators (e.g., serotonin, acetylcholine and dopamine). These neuromodulators are secreted by groups of neurons located in the basal forebrain and brainstem, and reach large regions of the brain, where they may act either via release from non-synapsing varicosities and extracellular diffusion or via synaptic junctions on specific neuronal populations.

The functional significance of the various types of overall human brain connectivity has been explored thanks to the development of neuroimaging and neurophysiological techniques as well as mathematical models. In particular, investigating the complete network of anatomically interconnected brain regions, the Connectome (Sporns et al., 2005), and its relationship with functional brain networks (using, for example, structural and functional MRI, magnetoencephalography and electroencephalography), has provided important advances in our knowledge of the general principles of structural and functional network organization of the human brain. In this regard, three types of connections are commonly recognized: (i) structural or anatomical connectivity, (ii) functional connectivity, defined as statistical associations or dependencies between neurophysiological events recorded in distant brain regions; and (iii) effective connectivity, defined as directed or causal relationships between brain regions (Bullmore and Sporns, 2009; Friston, 2011). Connectivity also evolves over time on multiple time scales (Galadí et al., 2021; Hansen et al., 2015) and establishes a functional connectivity dynamics predictive of aging (Battaglia et al., 2020; Escrichs et al., 2021), cognitive processes (Lombardo et al., 2020), and brain disease (Courtiol et al., 2020).

Neurons can be seen as central elements of a whole cascade of signal transduction, encompassing processes from the properties of ion channels up to the emergence of largescale activity states (Goldman et al., 2019). For example, the apical dendrites of pyramidal neurons integrate information from a large dendritic network, and may serve as gates or switches, enabling or breaking global brain dynamics and regulating information flow, therefore potentially having a central role in the mechanism of consciousness (Aru et al., 2020). According to this view, during conscious processing, the bottom-up information stream would be integrated at the apical dendrite with a top-down stream, putting into focus the role of large networks and cognitive processes.

On the largest scales, information processing capacity is characterized by the network's topochronic organization (Jirsa, 2008; Petkoski and Jirsa, 2021; Petkoski and Jirsa, 2019) as defined by the connectome's strength and signal transmission delays, constraining the emergence of brain functions, for instance, in the emergence of consciousness. The global neuronal workspace theory of consciousness is a concrete manifestation thereof and 
emphasizes the role of frontoparietal networks (Dehaene and Changeux, 2011). This theory is compared to other information-theory based (Tononi and Koch, 2015) and representational (Pennartz, 2015; Pennartz et al., 2019b), frameworks emphasizing the role of more posterior networks in, for instance, conscious vision, touch and hearing. Large-scale corticothalamic networks and the complexity of their dynamics play a major role in the levels of consciousness and their quantification, critical both for basic brain mechanistic understanding (Barbero-Castillo et al., 2021; Llinás et al., 1998; Sanchez-Vives et al., 2017) and for clinical application, as in disorders of consciousness (Comanducci et al., 2020; Demertzi et al., 2019; Storm et al., 2017). The topic of bottom-up vs. top-down perspectives in understanding multi-level brain organization has been intensively discussed in the past. It has been argued that a detailed bottom-up reconstruction and simulation of neuronal elements may reveal canonical microcircuits and reproduce results of in vivo experiments from which the laws of brain function will emerge (Markram et al., 2015). Along the same line of reasoning, it has been speculated that neuronal assemblies with their synaptic connections serve as innate, "Lego-like" building blocks of knowledge for perception and that the acquisition of memories involves the combination of these building blocks into complex constructs (Markram and Perin, 2011).

It is still a major challenge to explore how the different spatial scales are connected, for example, how precisely the binding of a neurotransmitter to its receptor modulates the activity of cell assemblies and large-scale networks involving long-distance fiber tracts and brain areas, from which, in the end, behavior emerges. Other questions are what the rules are that govern the underlying networks, and how it is possible that they are so effective and so efficient when they use so little energy. Likewise, much work remains to be done to elucidate how the brain interacts with the natural and cultural environment, e.g., how epigenetic mechanisms act on the brain, how genotype-phenotype relationships are linked with variations between brains and behavior, why aging or brain diseases affect some people more than others, and what determines the individual vulnerability to brain diseases.

Here, the top-down approach complements the strategy by using computational models as observation models that are fit to biological data (Friston, 2011; Huys et al., 2014; Pillai and Jirsa, 2017). These observational models effectively generate the data one would observe if the implicit generative model were correct. The explicit generative models establish a causal hypothesis, which uses the data to optimize the structure and parameters of some hypothetical network model, and evaluate the evidence for different models given the data. This dual approach guides the identification of causal mechanisms, going beyond the estimation of statistical correlations in traditional data mining approaches. Examples include the Perturbation Complexity Index $(\mathrm{PCl})$ used to assess effective connectivity (Comolatti et al., 2019), variants of dynamic causal modelling used in The Virtual Brain (TVB; see below for examples of clinical applications) and uses of generative models in a 'digital twin' approach (Hashemi et al., 2020; Vattikonda et al., 2021), which optimizes parameters to best explain personalized data as a prelude to characterizing within and between subject variability.

Many researchers converge on the notion that the two perspectives are not mutually exclusive and, even more, that bottom up-approaches need to be supplemented by conceptual approaches reducing structural complexity (Frégnac and Bathellier, 2015) and principled approaches making use of theories of brain function (Friston, 2011; Huys et al., 2014; Pillai and Jirsa, 2017). It has been argued to go beyond a simplistic top-down and bottom-up dichotomy, and to link the cognitive and brain perspectives (Ramsey and Ward, 2020). The unparalleled complexity of the brain may seem like a daunting challenge for any research project in the field, but it is a critical factor for the brain to organize itself and for the emergence of brain function and behavior. Cognition and behavior cannot be explained and predicted by the brain's individual components alone. Instead, both so-called bottomup and top-down approaches are necessary to understand brain organization, its role in signal transduction, cognitive processing and behavior. Information processing at axonal 
level is highly parallel, and at the same time characterized by both convergence and divergence (Rockland, 2020). It has been hypothesized that the laminar differentiation and the large number of neurons and areas, in combination with other factors, are key for cognitive abilities (Changeux et al., 2020; Pennartz et al., 2019a; Pennartz et al., 2019b).

Finally, a multiscale comprehensive understanding of cognitive function and behavior at the end requires not only to link the cellular with the cognitive perspective, but also to include intermediate levels of information processing such as areas and cortical columns. An example are columnar clusters in the human motion complex reflecting specific contents of consciousness (Schneider et al., 2019). Such clusters are components of the brain's organization into areas, layers, and other microstructural variations within areas (Amunts et al., 2020; Amunts and Zilles, 2015). Examples are giant Betz cells in the internal pyramidal cell layer of primary motor cortex, which give rise to long-range projections to the spinal cord, and the very broad and differentiated layer IV in the primary visual cortex, receiving massive input from the retina via the lateral geniculate body.

Thus, laminar patterns reflect connectivity (Rockland and DeFelipe, 2018), and suggest a specific role of an area in a network, e.g., underlying cognitive functions and consciousness (Goulas et al., 2018). The concept of the "localization of function" is more than 100 years old. It was inspired by early physiological and lesion studies such as pioneered by Broca (Broca, 1861), Campbell (Campbell, 1905), the Vogts (Vogt and Vogt, 1926) and Foerster (Foerster, 1934), which observed clinical symptoms, behavioral or brain activity changes, that were specific for a certain brain region. These studies were complemented by studies targeting disconnection syndromes, e.g. by Karl Wernicke, who studied brains with language deficit after brain lesion (Lichtheim, 1885; Wernicke, 1874). This concept integrates the network perspective with the perspective of brain regions critically involved in language, and proposed the first comprehensive theory of language. Structure-function relationships at the level of brain areas play an important role in modern neuroimaging, and are incorporated in recent concepts of brain segregation and integration (Eickhoff et

- Estimated number of nerve cells: about 86 billion, approximately the same number of glial cells, about 10.000 synapses per neuron. For comparison, a galaxy has about 100 billion stars.

- Type of signal transduction: electro-chemical with nerve conduction velocity between $1 \mathrm{~m} / \mathrm{s}$ to $100 \mathrm{~m} / \mathrm{s}$, while the speed of sound is about $343 \mathrm{~m} / \mathrm{s}$.

- Total length of connections: 2-3 million kilometers of fibers - for comparison, this is more than the diameter of the sun with 1.4 million kilometers

- Mass: 1200 - 1500 g, i.e., about $2 \%$ of the body weight

- Energy consumption: 20-30 Watt, i.e., about $20 \%$ of the total energy consumption of the body

al., 2018).

Box 1 The human brain in numbers and examples to illustrate their magnitudes

The comparison between species demonstrates that differences in brain organization are not simply a result of scaling as an effect of evolution, but are accompanied by changes in organization and complexity. A challenge results from the size of the human brain, and its increasing complexity. Major factors comprise, among others, the highly folded cerebral cortex, e.g., as compared to lisencephalic brains of rodents, the high degree of intersubject variability, and the large number of nerve cells, which is estimated to be 86 billion (Box 1, Fig. 1), as well as a greater molecular diversity of cell types (e.g., (Bakken et al., 
2021; Berg et al., 2021; Hodge et al., 2019)).

The large size of the human brain with its complex organization is reflected at the level of data that describe it (Box 2). While a digitized mouse brain with $1 \mu \mathrm{m}$ spatial resolution has a total volume of uncompressed data of 8 TBytes (Li et al., 2010), a similar model of the human brain, a 'digital twin' of its cellular structure, would be in the range of several PBytes. The interactive exploration (as opposed to simple storage and visualization) of such a dataset is beyond the capacities of current computing, and creates significant challenges in this field (Amunts and Lippert, 2021). Data coming from electron-microscopy, e.g., multi-beam electron-microscopy, result, for small samples at nanometer resolution, in comparable data sizes (Eberle and Zeidler, 2018).
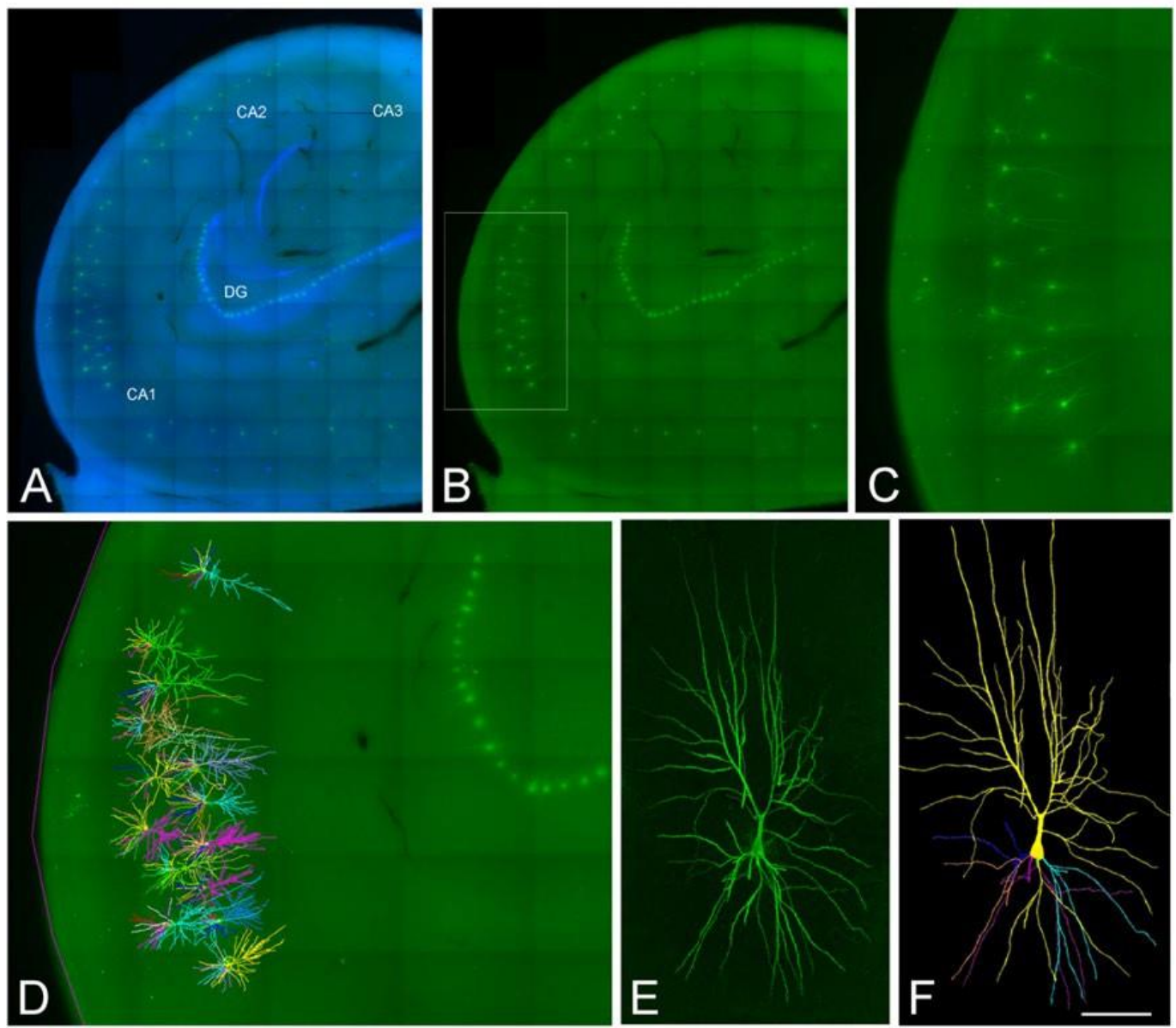

Fig. 1 Confocal microscopy images of human neurons injected with Lucifer yellow in the hippocampus. (A, B) Labeled pyramidal cells (green) and DAPI staining (blue) in different regions of the human hippocampus, including CA1, CA2, CA3 and the dentate gyrus region (DG). (C) Higher magnification image of the boxed region shown in $B$. (D) 3D reconstructed cells superimposed on the confocal image shown in C. (E, F) High-magnification image z projection showing an injected CA1 pyramidal cell $(E)$ and the 3D reconstruction of the same cell $(F)$. Scale bar $=1100 \mu \mathrm{m}$ in A, B; 460 $\mu \mathrm{m}$ in C, D; $100 \mu \mathrm{m}$ in E, F. Image taken from (Benavides-Piccione et al., 2019).

Big data problems also appear when moving from single brain data with high spatial or 
temporal resolution to large cohort studies with thousands of subjects, necessary to address intersubject variability. Large cohort studies are used to study the relationship of structural, functional, behavioral, life-style, health and genetic data in thousands of subjects, which are necessary to identify weak factors and their interactions in brain diseases. For example, the UK biobank provides a unique data set of about 500.000 participants (Bycroft et al., 2018). Neuroimaging PheWAS was recently introduced as a webbased system to analyze gene-brain relationships, and could be used to study the influences of the apolipoprotein E (APOE) gene on various brain morphological properties in the Alzheimer's Disease Neuroimaging Initiative (ADNI) cohort; benchmark tests on the UK biobank were performed as well (Zhao et al., 2020). The Human Connectome Projects has collected comprehensive neural data and tools, and set a standard in the field (Van Essen et al., 2013).

These and other examples highlight the increasing role of computing, web-based services and big data analytics in recent brain research. They also illustrate the relevance of largescale approaches, national and international consortia and research platforms, going beyond research at the level of single labs (Vogelstein et al., 2016). Technically, this is challenging as well: large storage and fast access, as well as powerful computers are required, including High-Performance Computing. Many applications also need most flexible regimes of work including interactive supercomputing and/or require to execute complex workflows (Amunts and Lippert, 2021). To organize research data in such a way that they are accessible, and well documented, while covering a large spectrum of spatial scales is still a challenge. High- quality solutions have been proposed for dedicated fields of application, e.g., Neurodata Without Borders (https://www.nwb.org/) for neurophysiological and morphological data at cellular level (Teeters et al., 2015). Another example are tissue models coming from the US BRAIN Initiative Cell Census Networks (BICCN; https://braininitiative.nih.gov/brain-programs/cell-census-network-biccn), which has started to publish very large data sets of small tissue pieces, but with ultra-high- resolution as cell reference atlases Callaway (Callaway et al., 2021). To integrate such information, coming from a multitude of labs, into their spatial, whole-brain context, however, is challenging at the computational and neuroinformatics side.

- $\quad$ An anatomical 3D model @ 1 micron resolution isotropic needs 2-3 PByte storage per brain

- $\quad$ To optimize the computation of fiber tracts with a spatial resolution of 60 microns isotropic would require years for the whole Human brain with current technology

Neuronal network training to extract structural features in images with a spatial resolution of $1 \times 1 \times 20$ microns would require, for the whole brain, 100 days at whole brain level with current technology

- A 10 seconds point-neuron simulation including 4 million neurons requires 10 minutes of computation on EBRAINS' Fenix system (400 core hours)

- One second of simulation of a network of 450,000 cells with a high level of details of the hippocampus CA1 region requires at least 20,000 cores and needs 130,000 core hours on the Piz Daint supercomputer at CSCS in Lugano, Switzerland.

- Simulation of the binding of a single substance at the molecular level with QM/MM (quantum mechanics/molecular mechanics): 20 million core hours on the JUWELS supercomputer at JSC, Germany.

Box 2 Estimated computational demands to study the human brain 


\section{The large-scale approach to advance neuroscience}

374 Accordingly, several large-scale approaches in brain research have been started to bundle 375 activities (Grillner, 2014). These approaches find a counterpart in other communities, e.g. 376 in the field of astrophysics or climate research, to name only a few of them. Different strategies have been chosen in the brain research community, e.g., addressing the "mind of the mouse" (Abbott et al., 2020), or to map structure and function of neuronal circuits by taking advantage of a non-human primate model, the common marmoset, as in Japan's Brain/MINDS project (Okano et al., 2015). The US BRAIN Initiative has an emphasis on the development of technologies to facilitate neuroscience research, and has just recently reported the generation of a cell census and atlas of the mammalian motor cortex; it is argued that a unified and mechanistic framework of neuronal cell type organization integrating multimodal molecular, genetic and spatial information has been established (Callaway et al., 2021). ENIGMA is a global alliance for "Enhancing Neurolmaging Genetics through Meta Analysis" (Thompson et al., 2020). The Human Connectome Projects is providing a large resource of data and tools to explore connectivity of the living human brain (http://www.humanconnectomeproject.org/ ), that is used worldwide as a basis of studies and experiments. These are only a few examples among several in this field. Comparable approaches can be found in other communities, e.g. biomolecular science (Elixir; https://elixir-europe.org/) and Covid-19 research (Research collaborations bring big rewards: the world needs more Nature 594, 301-302 (2021)), but also in other research fields such as particle physics (https://home.cern/). It has been argued that large-scale approaches are influential because they enable investigation of continuously arising new questions from the same data-rich sources and not because they answer any singlequestion (Abbott et al., 2020). At the same time, such approaches were, from their beginning, subject to controversy and criticism (Galison and Hevly, 1992; Mainen and Pouget, 2014).

Another argument for large-scale approaches comes from the high complexity of the research, requiring a collaborative effort over a long time-scale. This is true for research on the human brain. Its complexity, together with major progress in computing, motivated the researchers of the Human Brain Project (HBP, https://www.humanbrainproject.eu/en/) to initiate a large-scale research project in Europe (Markram et al., 2011). The HBP started in 2013 and was set up to get a deeper understanding of the brain in a time of breathtaking progress in computing and digital technologies (Amunts et al., 2016; Amunts et al., 2019; Markram et al., 2011). To achieve this aim, the HBP makes two major innovations: first, a new type of science creating synergy at the interface between empirical research on the brain and advanced computing, and second, an eco- system and new culture of collaboration leading to substantial progress in our understanding of the brain, brain medicine and brain-inspired technologies.

\section{EBRAINS research infrastructure}

Therefore, the HBP decided to develop a distributed, digital infrastructure, EBRAINS (https://ebrains.eu/ ). It is an open platform for researchers, offering technologically mature tools and services, which is permanently growing and expanding. While being built mainly by partners of the HBP, EBRAINS is increasingly serving the whole science community. It contains different tools and data, which can be combined and linked to each other in a flexible way, allowing researchers to solve their own research questions (Fig. 2). EBRAINS aims to become a powerful resource for the scientific community at large. Many elements of this infrastructure are already in place and can be accessed via its web portal.

EBRAINS is currently used and further developed to advance research mainly in three neuroscience area centered around connectivity: (i) Multiscale investigation of brain networks and connectivity, (ii) the role of networks in processes underlying cognition and consciousness, and (iii) artificial neural networks inspired by the brain, neurorobotics as 
well as neuromorphic processors, which serve both as accelerators for neuro-derived computation and as tools for neuroscience. A deeper understanding of how neural networks are built and how they function is a basic neuroscientific question of high relevance, and a prerequisite to achieve targeted interventions in brain disease and dysfunction, as well as to develop new diagnostic tools. The perspective of the brain as an embodied network also lets us draw inspiration for technology. New insights into the brain's information processing and network structure also provide a blueprint for research and development in neuromorphic computing and $\mathrm{Al}$, including deep learning, as well as neurorobotics.

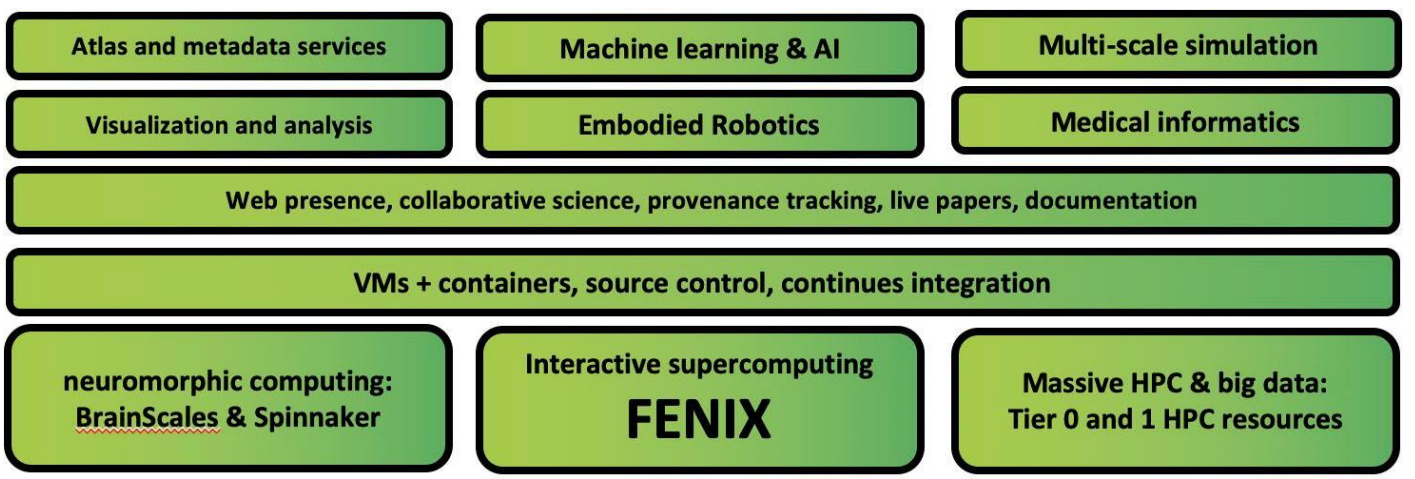

Fig. 2 The Human Brain Project' EBRAINS - a research infrastructure providing a broad set of tools and services which can be used to address challenges in brain research and brain-inspired technology (https://ebrains.eu/ ). The components can be combined resulting in special purpose solutions matching the different research challenges. EBRAINS is offering tools and services in the field of data \& knowledge (https://ebrains.eu/services/data-and-knowledge), atlases (https://ebrains.eu/services/atlases), simulation (https://ebrains.eu/services/simulation), brain-inspired technologies (https://ebrains.eu/services/brain-inspiredtechnologies), medical data analytics (https://ebrains.eu/services/medical-data) as well as a platform for collaboration (https://ebrains.eu/services/community).

Variations in structure and function between brains are a common thread running through research on connectivity at different spatial scales (Eickhoff et al., 2018; Finn et al., 2020; Larivière et al., 2019; Sun et al., 2016). Inter-subject variability can be observed in network organization, including the concentrations of individual receptors, functional connectivity as captured in fMRI, and structural connectivity at different levels. It expresses important properties of the brain linked to resilience against disease, and is an important target of research in itself, providing insights into brain organization (Zilles and Amunts, 2013). The degree to which brains may differ is linked to the genotype, changing during the whole life span and under conditions of brain diseases, e.g., (Caspers et al., 2014; Thompson et al., 2020). As a consequence, it is necessary for some research questions to study (very) large cohorts and 'Big Data' from neuroimaging, genetics, and behavior, to identify single factors and their interaction influencing the brain. The earlier mentioned UK biobank is an example of a very large cohort, and includes multimodal imaging data, sociodemographic, lifestyle and health-related information as well as a wide range of physical measures (Littlejohns et al., 2020).

A complementary strategy to consider inter-subject differences has been proposed in the context of the Individual Brain Charting Project (IBC), where spatial representations of multiple mental functions are targeted in a systematic and very comprehensive way in a small number of subjects; this also results in large data, because every subject is studied in depth, many times (Pinho et al., 2018). This data set is accessible through the Knowledge Graph and multilevel atlas of EBRAINS (Pinho et al., 2020; Pinho et al., 2021b), and can be analyzed in the context of other data sets that EBRAINS is hosting.

Such digital tools and platforms are functioning 'stand-alone', and often have an origin independent from the HBP. However, bringing them together under the roof of the EBRAINS 
research infrastructure opens up new avenues of application, increases their impact and makes their application more efficient (Fig. 2). This is feasible because EBRAINS is being developed collaboratively by neuroscientists and technology experts in a co- design approach for two reasons - to make sure that it fits the needs of neuroscientists, and to ensure that the platform is on a high technological maturity level, user-friendly, and professionally managed. It is also developed collaboratively with philosophers, ethicists, social scientists and public engagement experts, to build a research infrastructure with users that engage with and understand the ethical, philosophical and societal aspects of their work, and an infrastructure that is itself reliably, sustainably and responsibly constructed and managed.

EBRAINS offers different services (https://ebrains.eu/services/ ) for curating and sharing data and models, contributing to and accessing brain atlases, using modeling and simulation tools, running closed-loop Al and neurorobotics experiments, retrieving medical brain activity data, and computations based on high-performance computing. The idea behind this is to enable workflows that seamlessly connect elements of the different services. To prove this, so-called showcases have been developed by the HBP (Box 3).

Integrating brain data and knowledge from different research approaches requires curation, proper annotation and provenance tracking. Through the EBRAINS Knowledge Graph, a flexible and scalable metadata management system accompanied by a search user interface, data are made findable, accessible, interoperable and reusable, i.e., FAIR (Wilkinson et al., 2016). Knowledge graphs are powerful tools for community-based classification and data aggregation and are also being considered for use in other large brain projects (Yuste et al., 2020). A major challenge for developing a Knowledge Graph is that brain data are massive, complex, semantically and syntactically diverse, coming from many different studies. Accordingly, there is a great need for data and software standards to enable collaboration between scientists internationally (Abrams et al., 2021).
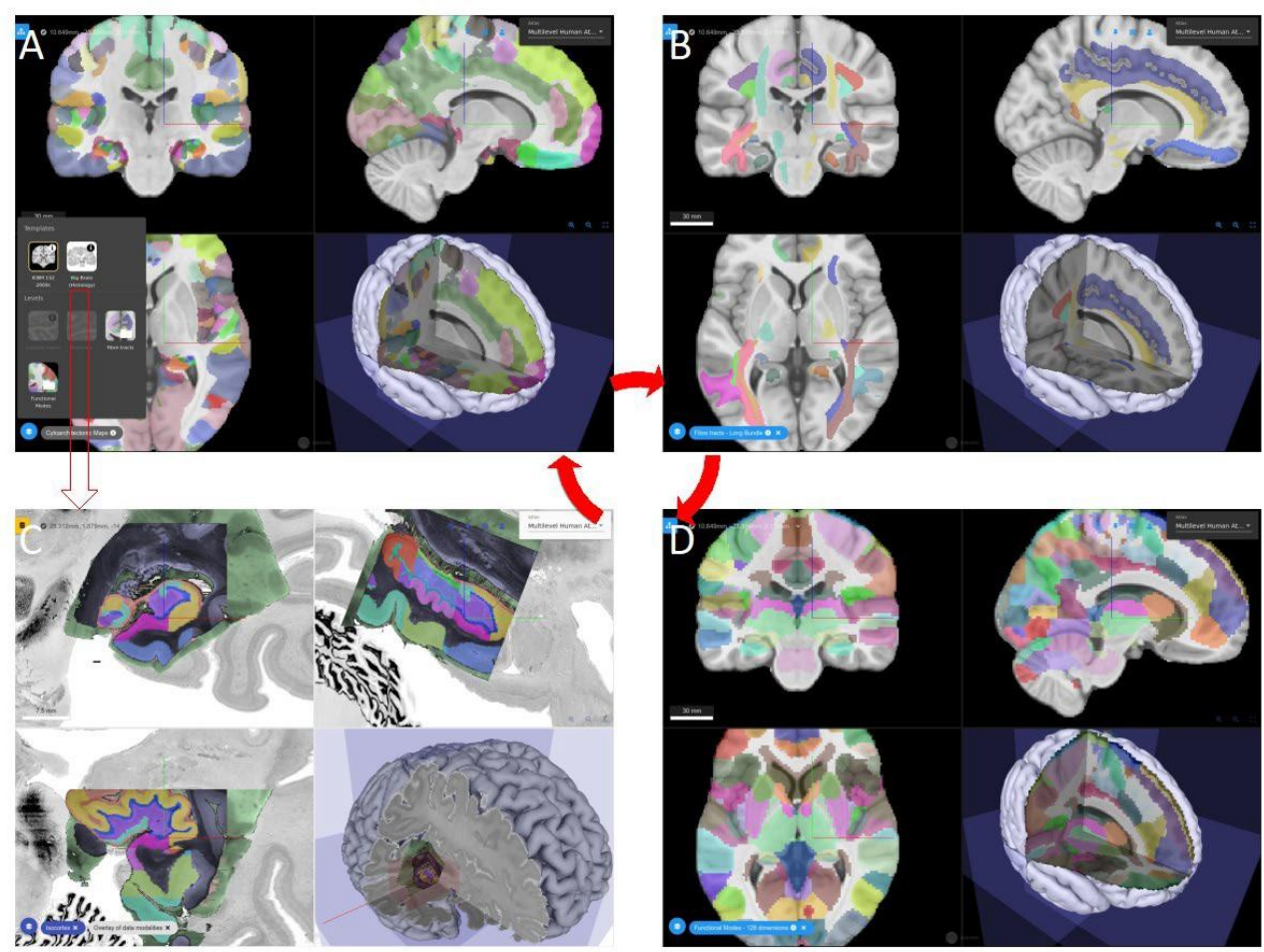
Fig. 3 The multi-level Human Brain Atlas provides different maps, e.g., (A) Julich-Brain cytoarchitectonic atlas (Amunts et al., 2020), (B) DTI-based maps of fiber bundles Guevara (Guevara et al., 2017; Guevara et al., 2012) and (D) functional parcellation based on task-based fMRI (Pinho et al., 2021a). (C) Microscopical data are available through the BigBrain model (Amunts et al., 2013). The atlas provides different types of data in a common spatial framework and allows switching between template spaces.

Brain atlases have a central role to visualize brain data in their spatial context, e.g., to interpret neuroimaging data from living human subject and patients, but also to derive therefrom input for subsequent analysis and model building. Comparative approaches targeting cross-species differences and similarities represent an important field of brain research, but there is still a gap in linking the atlases of the different brains under a common technological umbrella, which creates difficulties, e.g., in understanding homologies. The HBP human brain atlas aims to address this need, and to develop an atlas framework which allows reference to maps of human brain organization, those of rodents, and in the future also monkey brains. The atlas is comparable to "Google Earth", it allows zooming in and out, the visualization of regions of interest, data extraction from such regions, uploading new maps and results from the user's own research (Fig. 3). The BigBrain is an anatomical model at $20 \mu \mathrm{m}$ resolution (Amunts et al., 2013), allowing to map cellular information into a 3D reference space - from cortical layers (Wagstyl et al., 2020) and areas (Schiffer et al., 2021), to volume-of-interests integrated through the VoluBA atlastool (https://ebrains.eu/service/voluba/ ). The latter also opens the perspective to integrate data methods with subcellular resolution, including, e.g., those from electron microscopy, light sheet or two photon imaging. In addition, region-based data, e.g., from multiple receptors of neurotransmitters have been connected to cytoarchitectonically defined areas (Palomero-Gallagher and Zilles, 2019; Zilles and Amunts, 2009). The BigBrain is compatible to atlas data from neuroimaging, and serves as an input for simulation, e.g., using The Virtual Brain (see Showcase 1, Box 3).

Julich-Brain is a part of the Human Brain Atlas and serves as a cytoarchitectonic reference, while taking inter-subject variability into account (Amunts et al., 2020). It is linked to a comprehensive map of DTI-based fiber tracts (Guevara et al., 2017; Guevara et al., 2012), functional parcellation schemes based on multiple fMRI in a well-defined group of subjects (Pinho et al., 2021a), which provide insights into the cognitive dimension of brain parcellation. MR-based approaches are central to open up applications into in vivo imaging, which is relevant for medical research. Being on EBRAINS allows, for example, directly linking information from the atlases with models and simulation. In addition to a webbased viewers, python clients allow a fully programmatic software coupling, e.g., with simulation.

Simulation is increasingly enabled by the computational capabilities and capacities becoming available in Fenix (see below) to handle the very large data representing a human brain, and is in fact driving the development of computer science through its requirements. In the past few years, models of the cerebral cortex (Markram et al., 2015)), hippocampus (Coppolino et al., 2021), cerebellum (Casali et al., 2020), basal ganglia (Grillner and Robertson, 2016), typically at the cellular/circuit level, large-scale brain-simulations based on point neurons (Potjans and Diesmann, 2014) or mean-field network modelling (Goldman et al., 2021), as well as models of cognitive functions, such as spatial navigation (Coppolino et al., 2021), object recognition, scene understanding, visuo-motor functions, attention, perception and learning have been developed, and are being constantly improved.

Instead of performing a single simulation, targeted to "fit for everything", it became evident that various alternative approaches that complement each other, and are becoming more and more interlinked, are the way to proceed (Einevoll et al., 2019). The HBP has made available about 94 open-source models of neurons and brain circuits. They form reproducible building blocks for more large-scale integrated brain models. Related simulation engines (https://ebrains.eu/services/simulation/) allow the creation of a kind of "digital twins": from molecular to whole brain levels. Some models are directly linked to 
structural information from the brain atlases, and a first multi-level model of a human connectome, capturing connectivity of nerve cells, large-scale fiber tracts and functional neuronal networks, with underlying molecular, cellular and regional brain organization is under development. In parallel, there are also efforts towards cognitive models and (artificial) brain-inspired cognitive architectures are being constructed. Whereas in the past models aimed to reproduce either cognitive processes or physiological brain dynamics, current efforts are directed at models combining both dimensions: cognitive processing in dynamic brain architectures (Jaramillo et al., 2019).

Multilevel simulations for bridging several brain scales are currently realized by coupling simulators for different brain scales, such as single neurons or neuronal populations. Cosimulation technology now enables the synchronous simulation of bi-directionally coupled networks of firing-rate population models (e.g., in the TVB simulator) with regions of individual/networked neurons spiking models (e.g., in the NEST simulator; https://ebrains.eu/service/nest-simulator/). The coupling with other simulators (NEURON and Arbor; https://ebrains.eu/service/arbor/) is a topic of ongoing research.

It has been claimed simulation research represents the next phase of brain research (Fan and Markram, 2019). However, simulation efforts do not replace empirical research, but rather complement it. Ideally, a kind of cross-talk can be initiated, with simulation informing empirical research and vice-versa. For example, it layer 2/3 pyramidal neurons from the human temporal cortex have a membrane capacitance that was predicted by fitting in vitro voltage transients to theoretical transients and then validated by direct measurement in patch experiments (Eyal et al., 2016).

1. Degeneracy in neuroscience - when is Big Data big enough? Brains are maintaining full functionality within a range of normal variability. Finding out how and which structural changes affect (or not) brain function is an enormous computational challenge. Mastering this challenge will assist in the effort to deliver personalized brain medicine (Jirsa et al., 2017).

2. Improving epilepsy surgery with the Virtual BigBrain. "The Virtual Big Brain" aims to model and predict activity in an individual patient brain. It links data from high resolution brain mapping to brain avatars, running on high-performance computers to simulate the spread of individual seizure activity along cortical and subcortical surfaces (Proix et al., 2017).

3. Brain complexity and consciousness. Using new methods capable to differentiate states of consciousness from brain activity (Comolatti et al., 2019), and based on EBRAINS, brain simulations of sleep and wake modes have been created Goldman (Goldman et al., 2021). These simulations further the understanding of multiscale brain dynamics of different brain states towards individualized diagnosis and treatment, e.g., in unresponsive wakefulness or locked-in conditions.

4. Object perception and memory. To study perception, a brain-based perceptualcognitive architecture was integrated in a rodent-like robot. This architecture enables the robot to move around, navigate, remember, and find its way in simple environments. Due to its multisensory predictive coding model (Pearson et al., 2021), it shows enhanced place recognition capacity. These studies pave the way to create brain-inspired robots with perceptually enhanced navigation capabilities.

5. Dexterous in-hand object manipulation. Complex behaviors seem to be built on preexisting, simpler, building blocks ('motor primitives'). To investigate how they emerge, an anthropomorphic robotic hand is trained in several stages using a braininspired cognitive architecture. Increasingly complex actions are learned ultimately enabling the model to manipulate objects in the robotic hand. This approach bridges Al, neuroscience and robotics to help to explain why human brains learn skills with much less trials than standard artificial neural networks. 
Box 3: Showcases illustrating the applications of EBRAINS for neuroscientific research. All showcases rely on different elements of EBRAINS, and combine different approaches including simulation, robotics, atlasing, theory, data science and others. https://www.humanbrainproject.eu/en/science/highlights-and-achievements/

Simulation of human brain models is in most cases extremely compute intensive, and requires access to the most recent supercomputing resources. The Fenix infrastructure federates scalable storage and computing resources at multiple leading HPC sites in Europe in order to provide a single and readily available base infrastructure for data exchange and demanding computational tasks. On top of the Fenix infrastructure, any type of scientific digital service platform can be operated via RESTful APIs (https://fenix-ri.eu/ ). Fenix that emerged from computer science research in the HBP is an infrastructure- as-a-service (laaS) for EBRAINS. It has been developed to master the big data challenge of modern brain research. Generic-purpose and domain-specific services provide access to scalable and interactive computing resources via simple-to-use interfaces.

\section{Digital tools for diagnostics and treatments}

Understanding inter-subject variability in brain structure, connectivity and signal transduction on the one hand, and the factors modulating it at the different levels of brain organization on the other, is a central question for improving diagnostics and treatment of brain diseases, and key towards personalized brain medicine. Brain diseases represent a major challenge, not only for patients and their relatives, but also in terms of a burden for the health system and more generally, society (Box 4).

Mental, neurological and substance abuse disorders account for more than $10 \%$ of global

DALYs (DALY, or Disease-Adjusted Life Years, is a health metric calculated as the sum of

years of life lost and years lived with ). Six out of the ten disorders with disability highest

DALYs are related to the brain.

Brain diseases represent a considerable social and economic burden in Europe. With yearly costs of about 800 billion euros and an estimated 179 million (DiLuca and Olesen, 2014) people afflicted in 2010, brain diseases are an unquestionable emergency and a grand challenge for neuroscientists.

Epilepsy is one of the most common neurological disorders with an estimated prevalence of 50 million worldwide according to the World Health Organisation (2020). The complexity of the disease with its vast array of signs, symptoms, and underlying causes of seizures has been challenging to characterize, treat, and understand.

Worldwide, around 50 million people have dementia, with nearly 60\& living in low- and middle-income countries. Every year, there are nearly 10 million new cases. The total number of people with dementia is projected to reach 82 million in 2030 and 152 in 2050 (source WHO https://www.who.int/news-room/fact-sheets/detail/dementia).

Box 4 Brain Disorders and their relevance for society

Digital and computational tools are increasingly important in developing new diagnostic 
tools and options for therapy.

\section{The role of modeling and simulation in diagnosis and therapy}

Brain modeling and simulation play an increasing role in the development of new diagnostic and therapeutic solutions. Theoretical concepts built into simulation technologies such as The Virtual Brain (TVB; Fig. 4) (https://www.humanbrainproject.eu/en/medicine/thevirtual-brain/ ) allow the computation of patient-specific brain models serving as in-silico platforms for clinical hypothesis testing, improved diagnosis and development of novel interventions (Jirsa et al., 2017; Sanz-Leon et al., 2015). The generative brain models establish a causal hypothesis and are then evaluated against the patient's own brain imaging data (Friston et al., 2003; Jirsa et al., 2017). For instance, brain regions and fiber tracts serve as stimulation targets in TVB for the study of diagnostic and curative stimulation (Spiegler et al., 2016). 'Virtual surgery' can be performed mimicking a patient's actual surgery and simulating subsequent neural activity on the modified connectome, allowing the optimization of the efficiency of surgical interventions (An et al., 2019; Olmi et al., 2019) and the prediction of surgery outcomes (Aerts et al., 2020). The approach has also been applied to link molecular aspects of neurodegeneration in Alzheimer's disease with large-scale network modeling (Stefanovski et al., 2021). Modeling and simulation connect the advances in our understanding of brain function to a recent surge in the technological possibilities to write to and read from the brain, bringing together academic researchers, medical doctors and companies to expand the possibilities of linking digital technology to the nervous system and profoundly improve the lives of patients. It has recently been reported that researchers have developed a neuroprothesis for the blind, which was tested in monkeys (Chen et al., 2020). In this experimental study, monkeys were able to recognize different stimuli as simple shapes, motions or letters. The potential applications of brainmachine interfaces are expanding at a rapid pace, prompting the OECD "Science, Technology and Innovation Outlook" (OECD, 2016) to list neurotechnology as one of the ten most promising and disruptive future technologies.

Similarly, the HBP will increase the availability of integrated data and computational models supporting brain state transitions, network complexity and cognitive functions. The Perturbational Complexity Index ( $\mathrm{PCl})$ is a theory-inspired metric designed to gauge empirically the brain's capacity for integrating information (Comanducci et al., 2020). The $\mathrm{PCl}$ quantifies the algorithmic complexity (information) produced by the causal interactions that are triggered in the brain by a direct cortical perturbation. In practice $\mathrm{PCl}$ can be

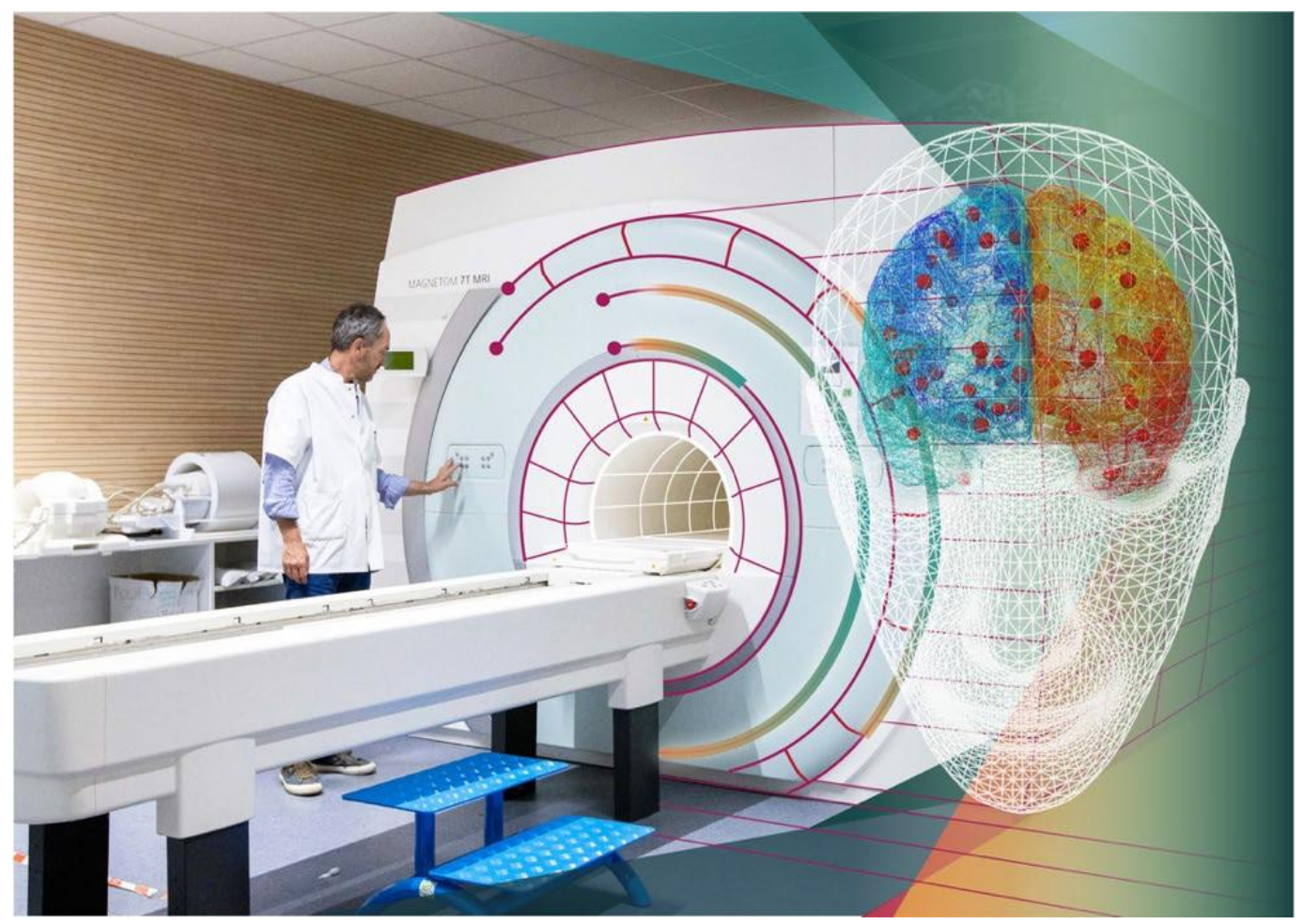


662

663

664

665

666

667

668

669

670

671

672

673

674

675

676

677

678

679

680

681

682

683

684

685

686

687

688

689

690

691

692

693

694

695

696

697

698

699

700

701

702

703

704

705

706

707

708

709

710

711

computed by compressing the overall brain electrophysiological response to a direct cortical perturbation with transcranial magnetic stimulation as well as by intracortical stimulation. I.e., the $\mathrm{PCl}$ is therefore another example illustrating how knowledge from basic neuroscience is informing theory and modeling, to be transferred into brain medicine.

Fig. 4 The Virtual Brain, a data driven neuroinformatics tool, fusing individual brain imaging data with atlas data and state-of-the-art brain modeling, for personalized simulations of brain activity and clinical interventions. Generative brain models operationalize a causal hypothesis, which is evaluated against the patient's own brain imaging data using variants of dynamical causal modeling such as Monte Carlo simulations (Hashemi et al., 2021; Hashemi et al., 2020; Sip et al., 2021; Vattikonda et al., 2021) (https://www.humanbrainproject.eu/en/medicine/the-virtual-brain/ ).

\section{The Medical Informatics Platform}

A Medical Informatics Platform (MIP; https://ebrains.eu/service/medical-informaticsplatform/) enables the analysis of large volumes of patient data throughout Europe (Redolfi et al., 2020). The MIP has opened the possibility to collect data from different hospitals, while considering high standards for data safety and security. It solves the data protection problem: locally installed software allows pooling of pre-analyzed data. These data can no longer be assigned to individual patients, but still provide valuable information. For diseases such as Alzheimer's and Parkinson's, this enables big-data and Al-driven approaches. Rare diseases with few cases per hospital can thus be analyzed in a statistically valid way. This could bring real breakthroughs, especially for this group, which together account for $20 \%$ of all brain diseases.

\section{The Human Intracerebral EEG Platform}

Human intracranial electroencephalographic (EEG) data describe brain dynamics with high temporal resolution, and provide unique insights into brain dynamics. At the same time, only a few centers derive such data from patients, and it is still difficult to integrate and analyze such patient data with sufficiently large numbers. The Human Intracerebral EEG Platform (HIP), together with analysis services, is being developed to capture such data (https://www.humanbrainproject.eu/en/medicine/human-intracerebral-eeg-platform/).

The idea behind is to pool such data from different sources. This will help to achieve a critical mass of valuable and unique patient data, to enable new clinical analyses based on large cohorts. It will also contribute to basic neuroscience research by providing insights into brain activity and its changes during cognitive tasks.

\section{Neuro-inspired technologies of EBRAINS}

Neuro-inspired technologies have a special position among research in the broader field of brain research as they are not only a tool to get new insights into the brain, but are also inspired by brain research to enable new technologies and computing. This includes (i) artificial neuronal networks and Al in general, (ii) neuromorphic computing, (iii) neurorobotics, as well as (iv) high-performance and modular supercomputing. The following paragraphs illustrate some examples.

\section{Artificial neuronal networks and Al}

Considerable progress has been made in implementing artificial neuronal networks, e.g., to classify (medical) images, and to produce in silico (cognitive) functions that are comparable to human cognitive functions. Recent progress is made also on applications that are more challenging to teach neural networks such as goal-directed planning, decision making and more general problem solving. The way artificial neuronal networks learn, however, currently differs significantly from the way we humans learn. Important aspects of learning in the human brain are not yet well understood, and new mechanisms of learning are 
712

discovered, which will further inform such approaches. Only recently, it has been shown that hippocampal output influences memory formation in the neocortex via sensory cortical layer 1 in rodents (Doron et al., 2020). It is expected that a systematic analysis of the differences and commonalities between artificial and natural networks will increasingly contribute to a better understanding of basic neuroscience and information processing, and result in improved concepts derived from large-scale and cellular networks in the brain.

New machine learning algorithms such as e-prop (short for e-propagation) use spikes in their model for communication between neurons in an artificial neural network. The cells only become active when their spikes are needed for information processing in the network. Learning is a particular challenge for such sparsely active networks, since longer observations are required to determine which neuron connections improve network performance. In addition, deep neural networks are by design well-tempered mathematical objects that allow back-propagation of error signals to drive learning through updates of synaptic weights, and spikes introduce discontinuities in neuronal dynamics that preclude the use of similar mathematical approaches (with some possible workarounds (Bellec et al., 2020; Zenke et al., 2021). Whether back-propagation itself is the right approach to capture the essential learning abilities of the human brain has long been an object of debate (Grossberg, 1988). E-prop now provides new solutions by means of a decentralized method, in which each neuron documents when its connections were used in a so-called e-trace (eligibility trace) (Bellec et al., 2020). It is speculated that e-prop will drive the development of a new generation of mobile learning computing systems that no longer need to be programmed but learn according to the model of the human brain and thus adapt to constantly changing requirements.

Methods have been proposed to further facilitate learning in recurrent, spiking neural networks, based on a target-based learning scheme in which the learning rule derived from likelihood maximization is used to mimic a specific spatio-temporal spike pattern that encodes the solution to complex temporal tasks (Muratore et al., 2021).

Highly detailed simulations of morphologically realistic, multi-compartment neuron models may also yield a unique perspective on the computational limitations of networks built on point neuron models (Gidon et al., 2020), and by extension, of all standard deep neural networks. A new study set out to find a computational method to make highly detailed models of neurons simpler, while retaining a high degree of realism (Wybo et al., 2021). It shows that (back-propagating) action potentials, $\mathrm{Ca}^{2+}$ spikes, and $\mathrm{N}$-methyl-D-aspartate spikes can all be reproduced with few compartments. The study also provides software that automates the simplification, to enable the inclusion of dendritic computations in network models.

In contrast with our everyday experience using brain circuits, it can take a prohibitively long time to train a computational system to produce the correct sequence of outputs in the presence of a series of inputs. By directly following the natural system's layout and circuitry of the hippocampus, models allow a level of efficiency and accuracy to be reached that opens the way to a new generation of learning architectures, including one shot learning (Coppolino et al., 2021).

The microcircuit of the cerebellum transforms internal signals implementing de facto computational algorithms that can be modified through learning. The discovery of adaptable transmission channels supports the long-sought spatiotemporal reconfiguration of the inputs that the cerebellum receives through its numerous sources. This turns into a multidimensional remapping of brain activity that allows the brain to learn from errors implementing sensorimotor and cognitive controllers, and to operate in a predictive manner. The new microcircuit properties are going to be implemented into large-scale models and inserted into closed-loop controllers, neurorobots, neuromorphic computers, and virtual brains, applicable to neuro-engineering, artificial intelligence, and neurology (Casali et al., 2020).

New computational approaches and models are being developed to underpin perception as 
765

a learning process in which the brain builds predictions and representations of what causes sensory inputs to arise the way they do (Pennartz et al., 2019a). Basic predictive coding approaches have been extended to large-scale, deep networks trained by Hebbian learning (Dora et al., 2021) have begun to integrate multiple sensory modalities (vision and touch) and have been made more neurobiologically realistic by implementing the principles in single-cell and spiking neural networks (Pearson et al., 2021).

\section{Neuromorphic Computing}

Synergies between advances in brain science and in neuromorphic, brain-inspired computing technologies are currently being explored, showing the potential of these technologies. The high energy consumption of artificial neural networks' learning activities is one of the biggest hurdles for the broad use of Artificial Intelligence in mobile applications. One approach to solve this problem can be gleaned from knowledge about the efficient transfer of information between neurons in the brain. Neurons send spikes to other neurons, but, to save energy, only as often as absolutely necessary.

Two complementary neuromorphic platforms are offered at EBRAINS as open services (https://ebrains.eu/service/neuromorphic-computing/ ):

SpiNNaker (Furber and Bogdan, 2020) supports very large-scale discrete time numerical simulation. Recent studies have shown that detailed simulations of the cortical microcircuit running on neuromorphic hardware (Fig. 5A) can outperform those on conventional machines, in terms of improved throughput and energy efficiency (Rhodes et al., 2020; van Albada et al., 2018).

BrainscaleS supports analogue continuous time accelerated emulation, compressing the time-scales required for long-term learning experiments by three to four orders of magnitude. Its modelling capabilities include structured neurons and active-dendrites (Aamir et al., 2018; Billaudelle et al., 2021).

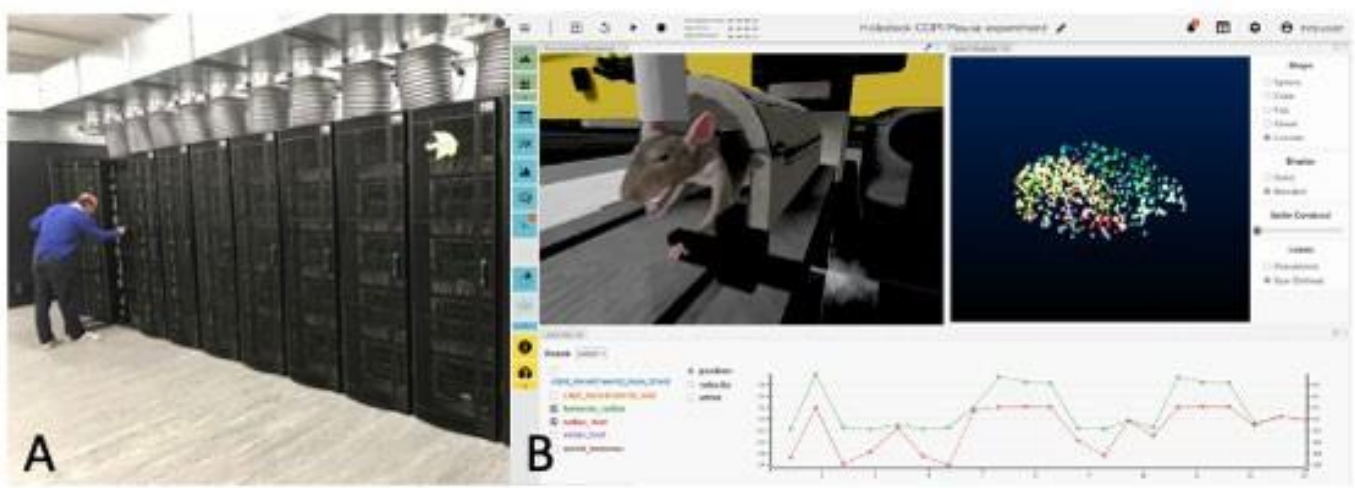

Fig. 5 Technologies driven by neuroscience. A The million-processor SpiNNaker machine at Manchester. B The user interface of the Neurorobotics Platform NRP, executing the virtualized copy of a real mouse experiment. The mouse body shown in the live rendering on the left is connected to a brain simulation that controls its muscle activations. Body movements are plotted in the graph at the bottom.

Neuromorphic technology is primed to converge with Al, offering much-needed perspectives in areas where the power demands of even the latest Al-specific chips limit their use at the edge to inference rather than learning. As such, EBRAINS services provides an opportunity for researchers working on this convergence, in the form of a toolchain that connects conceptual exploration to application prototyping and finally implementation. Edge 
802 computing applications are poised to benefit most from the emergence of neuromorphic chips capable of both energy-efficient, low-latency processing of data streams and concurrent learning based thereon. Autonomous robotics will also greatly benefit from such chips, insofar as they are in all likelihood key enabling technologies towards the implementation of complex cognitive functions such as decision-making, situational awareness, contextual adaptability, etc. Understanding how those arise from the human brain, both at the computational and implementation level, is a challenge taken on by the HBP.

\section{Neurorobotics}

Modeling how the brain is situated in a specific environment with which it interacts through its body is mandatory for understanding how neural activity and physical behavior give rise to each other. In line with the position of enactivism, embodied modeling of perception and cognition stresses that actions of the body endow the brain with causal power in the world and that any neuronal network likely serves the purpose (directly or indirectly) to enhance successful interaction with a complex, dynamic, environment. Neurorobotics provides both the tools and the theory for embedding brain simulations into robotic bodies to establish a closed loop of perception, cognition and action between the brain, its body and the environment (Fig. 5B). This makes it possible to not only create highly detailed models of the brain's structure but to also reproduce the dynamics that emerge from them under highly realistic conditions.

The Neurorobotics Platform (https: //neurorobotics.net/) of the HBP (Falotico et al., 2017) provides an integrated cloud-based simulation framework for the design and execution of virtual neurorobotics experiments in physically realistic environment models (Fig. 3B). The platform is able to run large-scale spiking neuronal networks implemented with the NEST simulator on supercomputers on the order of millions of neurons, billions of synapses (Helias et al., 2012)), and supports modular, heterogeneous control architectures for the simulated agents. It is also accessible via https://ebrains.eu/service/neuroroboticsplatform/.

As the Neurorobotics Platform contains simulation models and tools required to replace all components of traditional neuroscience experiments by digital twins, it lays the foundations for virtualized neuroscience. Fully virtual experiments cannot only reproduce previously achieved findings from the lab but importantly also predict new results at high speed and low cost. The more these predictions are refined by subsequent experimental ground truth, the better future predictions get. This makes research not only more efficient but considerably enlarges the exploration space.

Another major advantage of virtual neuroscience is that the full state of the experiment from the activations of muscles to the firing of individual neurons is observable any time at any desired level of detail. This enables a new form of real-time brain atlases where not only the brain's structure can be observed but also its live activity. These atlases therefore not only represent space but also time.

Closed-loop neurorobotic systems are not constrained to virtual experiments. They can also be set up in the real world by connecting a brain simulation to a physical robot. In particular, neurorobotics allows for embodiment of cognitive architectures on anthropomorphic robots thus enabling the transfer of emulated human capacities to artificial agents. The adaptive "brains" of these robotic agents are amenable to close scrutiny, and inspecting how they solve goal-directed tasks may inspire new testable hypotheses whether the human brain has developed similar representations and processes (Kroner et al., 2020). Neuromorphic computing is an essential prerequisite for these studies because the simulation of the neural models needs to run in real-time. This makes neurorobotics an ideal tool to prototype applications that embed neuromorphic computing at their core, but also rely on complementary, more standard technologies. Such prototyping is made all the easier by the fact that the Neurorobotics Platform can natively use neuromorphic hardware as a simulation backend and will also be enabled in the future to perform hardware-in-the-loop simulations. 
Building adaptive biologically inspired cognitive architectures contributes to our understanding how the brain works by emulating some aspects of its functions. For example, large-scale neural network models are created that are themselves composed of smaller neural network modules that correspond roughly to specific brain areas. These types of architectures enable the development of new types of training protocols and the investigation of long-standing questions such as the separation problem and the binding problem (von der Malsburg, 1999). Neurorobotics therefore not only provides the foundations for virtual neuroscience but also enables effective knowledge transfer to artificial intelligence and machine learning.

\section{High-performance and modular supercomputing}

While neuroscience in the past rather rarely required extreme-scale computing, the need to simulate at large scale or to process and analyze data sets in the PByte range has changed the situation (e.g., (Amunts et al., 2014; Amunts and Lippert, 2021; Einevoll et al., 2019; Franceschini et al., 2020; Menzel et al., 2019; Rossetti et al., 2019)) and motivated the development of the federated Europe-wide HPC infrastructure Fenix (https://fenix-ri.eu/ ). Meanwhile, a strong community has emerged to drive such development, and Fenix resources are openly available for compute and storage intensive projects. The methods that are being developed in this context often go beyond neuroscience, and are open to other research communities. Both edge computing and cloud computing are considered for use cases from neuroscience. The HBP is developing tools for interactive supercomputing, web-based visualization and analysis of big data in the context of Fenix. Researchers are preparing use-cases for Exascale performance on modular supercomputers to be built in 2023/24 under the umbrella of the EuroHPC Joint Undertaking and participating countries to coordinate their efforts and pool their resources in Europe to enable world-class Exascale supercomputers, together with researchers from other communities. Joint interests in the development of high-performance computing, its hardware and software, will open new perspectives for collaborative project across different research domains.

\section{Collaborative perspectives}

In the middle and long run, the aim is to further develop EBRAINS as a global platform for collaboration and exchange among researchers, a mechanism for users to participate in the development of new tools, methods, and to provide and exchange their data. Such digital research infrastructure is not only relevant for individual collaboration between researchers, but also between large-scale initiatives, e.g., the US BRAIN Initiative, with initiatives such as Healthy Brains for Healthy Lives (HBHL) in Canada, and brain initiatives in China, Japan, Australia, to name some of them. For example, the Canadian-German collaboration HIBALL (https: //bigbrainproject.org/hiball.html ) focuses on the BigBrain as a high-resolution model of the human brain (Amunts et al., 2013) to reinforce utilization and co-development of the latest $\mathrm{Al}$ and high-performance computing technologies for building highly detailed 3D brain models, and connects EBRAINS and HBHL. It provides next-generation brain models, integrates multimodal data to the BigBrain, takes care about interoperability of scientific workflows, and develops new deep neural network architectures. It has built an active community in a short time that uses and further develops tools for brain research. Such synergy became feasible also because it can build upon existing infrastructures both in Canada and Europe. It would also be a tool that can be used to link ultra-high-resolution models of volume of interest such as developed in the BRAIN Initiative Cell Census Network, e.g., from the primary motor cortex (Callaway et al., 2021). This would have the advantage of integrating highly detailed, multimodal information into its spatial context, thereby linking advantages of the bottom-up with the top-down approach.

Several brain initiatives have founded the International Brain Initiative (IBI; 
https://www.internationalbraininitiative.org/ ) to join forces. As an integral part of the science and technology agenda, IBI addresses questions of ethics, philosophy and society. Specifically, at the interface of neuroscience and technology, the clinic and society, new challenging issues arise, including, for example, data protection and privacy, pharmacological and digital neuroenhancement, and dual use of brain-related technologies (Flick et al., 2020; Salles et al., 2019a). Another new field is concerning the ethics of Al, which plays an increasing role (Stahl, 2021). All these questions have in common that they cannot be answered by a single discipline, but require a cross-disciplinary interaction and broader discussion in society. Technical advances need to be delivered in a way that reflects European values and principles, such as non-discrimination, fairness and privacy. Ethical considerations like these are an integral part of technology developments in EBRAINS. Through the efforts of the Human Brain Project, EBRAINS is intended to integrate neuroethics and philosophical analysis to enhance the neuroscientific work (Evers, 2009; Salles et al., 2019a; Salles et al., 2019b). Philosophical analysis provides clarification of scientific concepts such as behavior, intelligence, digital twin and consciousness and explores how neuroscientific knowledge is constructed, what are its underlying assumptions and how they are justified, how results may be interpreted, and why or how empirical knowledge of the brain can be relevant to philosophical, social, and ethical concerns (Pennartz, 2015; Salles et al., 2019b).

Conceptual clarification and analysis are the basis for addressing more practical issues raised by neuroscientific research from data protection autonomy and identity concerns (Amadio et al., 2018)). EBRAINS is expected to adopt an inclusive and co-creative way of working, engaging with multiple audiences and communities to discuss ethical issues, developing novel insights into responsible innovations and their clinical and societal applications (https://ebrains.eu/discover/ ).

\section{Conclusions}

To achieve a comprehensive understanding of the human brain, its connectome and parcellations means understanding the multi-level organization of the brain as an embodied network and complex system enabling perception, action, consciousness and cognition. Combining the perspectives of multi-level brain organization with embodiment is not only relevant to capture the full scope of brain diseases and to be able to develop new therapies, but also for the development of neuro-inspired technologies, and future neurorobotics.

There is an urgent need to accelerate efforts for mental and brain health by making full use of insights from brain research and modern digital tools. Based on use cases from neurology already available in EBRAINS, including the Medical Informatics Platform and the Human Intracerebral EEG Data Platform, it is now being further developed to support research in mental health, psychiatric disorders, neurosurgery, and neuroradiology, but also more broadly in the medical field.

Insights into fundamental questions of brain organization will provide the key to new computing technologies, artificial neuronal networks, cognitive computing and neurorobotics as an integrative overarching technology both for experimentation and for substantially advancing real robotics. Making such technologies more "neuro-inspired" is expected to significantly speed up their development. Neurorobotics and neuromorphic computing will benefit from being increasingly neuro-inspired.

The amount of brain data is increasing rapidly. The effort in terms of time, knowledge and methodology needed to make it findable, accessible, interoperable and reusable (FAIR) has long been underestimated and resources should be planned, from the very beginning of each research project, to address this.

The Human Brain Atlas allows access to multiple brain data according to their spatial 
organization through viewers, but also fully programmed software coupling. This might bea game changer for analyses of big and complex data on systems of the highest performance, but also for modeling and simulation, which become biologically more realistic.

Modeling and simulation have started to develop from different angles, and they used different approaches. But now we are in a position where we can link them, which enables bridging the different scales, to better constrain and to verify results of simulation.

Collaboration across boundaries of institutions, sectors, nations, research disciplines and cultures is indispensable for progress in neuroscience. Moreover, insights from brain research will increasingly influence learning and education and have an impact on our society.

To stay ahead of emerging ethical, societal and legal issues, and to strengthen the societal benefit and acceptability of its findings, EBRAINS need structures and strategies for engaging in dialogue with communities on issues of immediate and long-term relevance, including data ethics, neuroethics, animal use and well-being, dual use, gender equality and diversity.

The culture of collaboration in the neurosciences is changing. The authors are convinced that we can contribute to making it more open, cooperative and participatory, for the benefit of neuroscience, medicine and society, which marks the beginning of a new paradigm to understand the brain.

\section{References}

Aamir, S.A., Müller, P., Kiene, G., Kriener, L., Stradmann, Y., Grübl, A., Schemmel, J., and Meier, K. (2018). A Mixed-Signal Structured AdEx Neuron for Accelerated Neuromorphic Cores. IEEE Transactions on Biomedical Circuits and Systems 12, 1027-1037. 10.1109/TBCAS.2018.2848203.

Abbott, L.F., Bock, D.D., Callaway, E.M., Denk, W., Dulac, C., Fairhall, A.L., Fiete, I., Harris, K.M., Helmstaedter, M., Jain, V., et al. (2020). The Mind of a Mouse. Cell 182, 1372-1376. 10.1016/j.cell.2020.08.010.

Abrams, M.B., Bjaalie, J.G., Das, S., Egan, G.F., Ghosh, S.S., Goscinski, W.J., Grethe, J.S., Kotaleski, J.H., Ho, E.T.W., Kennedy, D.N., et al. (2021). A Standards Organization for Open and FAIR Neuroscience: the International Neuroinformatics Coordinating Facility. Neuroinformatics, 10.1007/s12021-12020-09509-12020. 10.1007/s12021-020-09509-0.

Adams, A., Albin, S., Amunts, K., Asakawa, T., Bernard, A., Bjaalie, J.G., Chakli, K., Deshler, J.O., De Koninck, Y., Ebell, C.J., et al. (2020). International Brain Initiative: An Innovative Framework for Coordinated Global Brain Research Efforts. Neuron 105, 212-216. 10.1016/j.neuron.2020.01.002.

Aerts, H., Schirner, M., Dhollander, T., Jeurissen, B., Achten, E., Van Roost, D., Ritter, P., and Marinazzo, D. (2020). Modeling brain dynamics after tumor resection using The Virtual Brain. Neuroimage 213, 116738. 10.1016/j.neuroimage.2020.116738.

Amadio, J., Bi, G.-Q., Boshears, P.F., Carter, A., Devor, A., Doya, K., Garden, H., Illes, J., Johnson, L.S.M., Jorgenson, L., et al. (2018). Neuroethics Questions to Guide Ethical Research in the International Brain Initiatives. Neuron 100, 19-36. 10.1016/j.neuron.2018.09.021.

Amunts, K., Axer, H., and Bücker, O. (2014). Towards a multi-scale, high-resolution model of the human brain. In Brain-Inspired Computing, L. Grandinetti, N. Petkov, and T. Lippert, eds. (Springer International Publishing Switzerland), pp. 3-14.

Amunts, K., Ebell, C., Muller, J., Telefont, M., Knoll, A., and Lippert, T. (2016). The Human Brain Project: Creating a European Research Infrastructure to Decode the Human Brain. Neuron 92, 574-581. 10.1016/j.neuron.2016.10.046.

Amunts, K., Knoll, A., Lippert, T., Pennartz, C.M., Ryvlin, P., Destexhe, A., Jirsa, V.K., 
1008

1009

1010

1011

1012

1013

1014

1015

1016

1017

1018

1019

1020

1021

1022

1023

1024

1025

1026

1027

1028

1029

1030

1031

1032

1033

1034

1035

1036

1037

1038

1039

1040

1041

1042

1043

1044

1045

1046

1047

1048

1049

1050

1051

1052

1053

1054

1055

1056

1057

1058

1059

1060

1061

1062
D'Angelo, E., and Bjaalie, J.G. (2019). The Human Brain Project - synergy between neuroscience, computing, informatics and brain inspired technologies. PLoS Biol 17(7):e3000344.

Amunts, K., Lepage, C., Borgeat, L., Mohlberg, H., Dickscheid, T., Rousseau, M.E., Bludau, S., Bazin, P.L., Lewis, L.B., Oros-Peusquens, A.M., et al. (2013). BigBrain: An ultrahighresolution 3D human brain model. Science 340, 1472-1475.

Amunts, K., and Lippert, T. (2021). Brain research challenges supercomputing. Science 374, 1054-1055. doi:10.1126/science.abl8519.

Amunts, K., Mohlberg, H., Bludau, S., and Zilles, K. (2020). Julich-Brain: A 3D probabilistic atlas of the human brain's cytoarchitecture. Science 369, 988-992. $10.1126 /$ science.abb4588.

Amunts, K., and Zilles, K. (2015). Architectonic mapping of the human brain beyond Brodmann. Neuron 88, 1086-1107.

An, S., Bartolomei, F., Guye, M., and Jirsa, V. (2019). Optimization of surgical intervention outside the epileptogenic zone in the Virtual Epileptic Patient (VEP). PLoS Comput Biol 15, e1007051. 10.1371/journal.pcbi.1007051.

Aru, J., Suzuki, M., and Larkum, M.E. (2020). Cellular Mechanisms of Conscious Processing. TINS 24, 814-825. https://doi.org/10.1016/j.tics.2020.07.006.

Bakken, T.E., Jorstad, N.L., Hu, Q., Lake, B.B., Tian, W., Kalmbach, B.E., Crow, M., Hodge, R.D., Krienen, F.M., Sorensen, S.A., et al. (2021). Comparative cellular analysis of motor cortex in human, marmoset and mouse. Nature 598, 111-119. 10.1038/s41586-02103465-8.

Barbero-Castillo, A., Mateos-Aparicio, P., Dalla Porta, L., Camassa, A., Perez-Mendez, L., and Sanchez-Vives, M.V. (2021). Impact of $G A B A<$ sub $>A</$ subs $>$ and $G A B A<$ sub $>B</$ sub $>$ Inhibition on Cortical Dynamics and Perturbational Complexity during Synchronous and Desynchronized States. The Journal of Neuroscience 41, 5029-5044. 10.1523/jneurosci.1837-20.2021.

Battaglia, D., Boudou, T., Hansen, E.C.A., Lombardo, D., Chettouf, S., Daffertshofer, A., McIntosh, A.R., Zimmermann, J., Ritter, P., and Jirsa, V. (2020). Dynamic Functional Connectivity between order and randomness and its evolution across the human adult lifespan. Neuroimage 222, 117156. https://doi.org/10.1016/j.neuroimage.2020.117156.

Bellec, G., Scherr, F., Subramoney, A., Hajek, E., Salaj, D., Legenstein, R., and Maass, W. (2020). A solution to the learning dilemma for recurrent networks of spiking neurons. Nature communications 11, 3625. 10.1038/s41467-020-17236-y.

Benavides-Piccione, R., Regalado-Reyes, M., Fernaud-Espinosa, I., Kastanauskaite, A., Tapia-González, S., León-Espinosa, G., Rojo, C., Insausti, R., Segev, I., and DeFelipe, J. (2019). Differential Structure of Hippocampal CA1 Pyramidal Neurons in the Human and Mouse. Cerebral Cortex 30, 730-752. 10.1093/cercor/bhz122.

Berg, J., Sorensen, S.A., Ting, J.T., Miller, J.A., Chartrand, T., Buchin, A., Bakken, T.E., Budzillo, A., Dee, N., Ding, S.L., et al. (2021). Human neocortical expansion involves glutamatergic neuron diversification. Nature 598, 151-158. 10.1038/s41586-021-03813-8.

Billaudelle, S., Cramer, B., Petrovici, M., Schreiber, K., Kappel, D., Schemmel, J., and Meier, K. (2021). Structural plasticity on an accelerated analog neuromorphic hardware system. Neural Networks 133, 11-20. 10.1016/j.neunet.2020.09.024.

Broca, P. (1861). Remarques sur le si,ge de la facult, du langage articul,, suivies d'une observation d'aphemie (Perte de la Parole). Bulletins et Memoires de la Societe Anatomique de Paris 36, 330-357.

Bullmore, E., and Sporns, O. (2009). Complex brain networks: graph theoretical analysis of structural and functional systems. Nature Reviews Neuroscience 10, 186-198.

Bycroft, C., Freeman, C., Petkova, D., Band, G., Elliott, L.T., Sharp, K., Motyer, A., Vukcevic, D., Delaneau, O., O'Connell, J., et al. (2018). The UK Biobank resource with deep phenotyping and genomic data. Nature 562, 203-209. 10.1038/s41586-018-0579-z.

Callaway, E.M., Dong, H.-W., Ecker, J.R., Hawrylycz, M.J., Huang, Z.J., Lein, E.S., Ngai, J., Osten, P., Ren, B., Tolias, A.S., et al. (2021). A multimodal cell census and atlas of the mammalian primary motor cortex. Nature 598, 86-102. 10.1038/s41586-021-03950-0. 
1063 Campbell, A.W. (1905). Histological Studies on the Localisation of Cerebral Function 1064 (Cambridge University Press).

1065 Casali, S., Tognolina, M., Gandolfi, D., Mapelli, J., and D'Angelo, E. (2020). Cellular1066 resolution mapping uncovers spatial adaptive filtering at the rat cerebellum input stage. 1067 Communications Biology 3, 635. 10.1038/s42003-020-01360-y.

1068 Caspers, S., Moebus, S., Lux, S., Pundt, N., Schütz, H., Mühleisen, T.W., Gras, V., 1069 Eickhoff, S.B., Romanzetti, S., Stöcker, T., et al. (2014). Studying variability in human 1070 brain aging in a population-based German cohort-rationale and design of 1000BRAINS. 1071 Front. Aging Neurosci. 6, 149. 10.3389/fnagi.2014.00149.

1072 Changeux, J.-P., Goulas, A., and Hilgetag, C.C. (2020). A Connectomic Hypothesis for the 1073 Hominization of the Brain. Cerebral Cortex 31, 2425-2449. 10.1093/cercor/bhaa365.

Chen, X., Wang, F., Fernandez, E., and Roelfsema, P.R. (2020). Shape perception via a high-channel-count neuroprosthesis in monkey visual cortex. Science 370, 1191-1196. $10.1126 /$ science.abd7435.

Comanducci, A., Boly, M., Claassen, J., De Lucia, M., Gibson, R.M., Juan, E., Laureys, S., Naccache, L., Owen, A.M., Rosanova, M., et al. (2020). Clinical and advanced neurophysiology in the prognostic and diagnostic evaluation of disorders of consciousness: review of an IFCN-endorsed expert group. Clinical Neurophysiology 131, 2736-2765. https://doi.org/10.1016/j.clinph.2020.07.015.

Comolatti, R., Pigorini, A., Casarotto, S., Fecchio, M., Faria, G., Sarasso, S., Rosanova, M., Gosseries, O., Boly, M., Bodart, O., et al. (2019). A fast and general method to empirically estimate the complexity of brain responses to transcranial and intracranial stimulations. Brain Stimul 12, 1280-1289. 10.1016/j.brs.2019.05.013.

Coppolino, S., Giacopelli, G., and Migliore, M. (2021). Sequence Learning in a Single Trial: A Spiking Neurons Model Based on Hippocampal Circuitry. IEEE Trans Neural Netw Learn Syst Pp. 10.1109/tnnls.2021.3049281.

Courtiol, J., Guye, M., Bartolomei, F., Petkoski, S., and Jirsa, V.K. (2020). Dynamical Mechanisms of Interictal Resting-State Functional Connectivity in Epilepsy. J. Neurosci. 40, 5572-5588. 10.1523/jneurosci.0905-19.2020.

Dehaene, S., and Changeux, J.P. (2011). Experimental and theoretical approaches to conscious processing. Neuron 70, 200-227.

Demertzi, A., Tagliazucchi, E., Dehaene, S., Deco, G., Barttfeld, P., Raimondo, F., Martial, C., Fernández-Espejo, D., Rohaut, B., Voss, H.U., et al. (2019). Human consciousness is supported by dynamic complex patterns of brain signal coordination. Science Advances 5 , eaat7603. 10.1126/sciadv.aat7603.

DiLuca, M., and Olesen, J. (2014). The cost of brain diseases: a burden or a challenge? Neuron 82, 1205-1208. 10.1016/j.neuron.2014.05.044.

Dora, S., Bohte, S.M., and Pennartz, C.M.A. (2021). Deep Gated Hebbian Predictive Coding Accounts for Emergence of Complex Neural Response Properties Along the Visual Cortical Hierarchy. Front. Comput. Neurosci. 15, 666131. 10.3389/fncom.2021.666131.

Doron, G., Shin, J.N., Takahashi, N., Drüke, M., Bocklisch, C., Skenderi, S., de Mont, L., Toumazou, M., Ledderose, J., Brecht, M., et al. (2020). Perirhinal input to neocortical layer 1 controls learning. Science 370. 10.1126/science.aaz3136.

Eberle, A.L., and Zeidler, D. (2018). Multi-Beam Scanning Electron Microscopy for HighThroughput Imaging in Connectomics Research. Frontiers in Neuroanatomy 12. 10.3389/fnana.2018.00112.

Eickhoff, S.B., Yeo, B.T.T., and Genon, S. (2018). Imaging-based parcellations of the human brain. Nat. Rev. Neurosci. 19, 672-686. 10.1038/s41583-018-0071-7.

Einevoll, G.T., Destexhe, A., Diesmann, M., Grun, S., Jirsa, V., de Kamps, M., Migliore, M., Ness, T.V., Plesser, H.E., and Schurmann, F. (2019). The Scientific Case for Brain Simulations. Neuron 102, 735-744. 10.1016/j.neuron.2019.03.027.

Escrichs, A., Biarnes, C., Garre-Olmo, J., Fernández-Real, J.M., Ramos, R., Pamplona, R., Brugada, R., Serena, J., Ramió-Torrentà, L., Coll-De-Tuero, G., et al. (2021). Whole-Brain Dynamics in Aging: Disruptions in Functional Connectivity and the Role of the Rich Club. Cereb. Cortex 31, 2466-2481. 10.1093/cercor/bhaa367. 
1118

1119

1120

1121

1122

1123

1124

1125

1126

1127

1128

1129

1130

1131

1132

1133

1134

1135

1136

1137

1138

1139

1140

1141

1142

1143

1144

1145

1146

1147

1148

1149

1150

1151

1152

1153

1154

1155

1156

1157

1158

1159

1160

1161

1162

1163

1164

1165

1166

1167

1168

1169

1170

1171

1172

Evers, K. (2009). Neuroéthique - Quand la matière s'éveille La lettre du Collège de France La Lettre $n^{\circ} 25$.

Eyal, G., Verhoog, M.B., Testa-Silva, G., Deitcher, Y., Lodder, J.C., Benavides-Piccione, R., Morales, J., DeFelipe, J., de Kock, C.P.J., Mansvelder, H.D., and Segev, I. (2016). Unique membrane properties and enhanced signal processing in human neocortical neurons. eLife 5, e16553. 10.7554/eLife.16553.

Falotico, E., Vannucci, L., Ambrosano, A., Albanese, U., Ulbrich, S., Vasquez Tieck, J.C., Hinkel, G., Kaiser, J., Peric, I., Denninger, O., et al. (2017). Connecting Artificial Brains to Robots in a Comprehensive Simulation Framework: The Neurorobotics Platform. Front. Neurorobot. 11, 2. 10.3389/fnbot.2017.00002.

Fan, X., and Markram, H. (2019). A Brief History of Simulation Neuroscience. Frontiers in Neuroinformatics 13. 10.3389/fninf.2019.00032.

Finn, E.S., Glerean, E., Khojandi, A.Y., Nielson, D., Molfese, P.J., Handwerker, D.A., and Bandettini, P.A. (2020). Idiosynchrony: From shared responses to individual differences during naturalistic neuroimaging. Neuroimage 215, 116828. 10.1016/j.neuroimage.2020.116828.

Flick, C., Zamani, E.D., Stahl, B.C., and Brem, A. (2020). The future of ICT for health and ageing: Unveiling ethical and social issues through horizon scanning foresight. Technological Forecasting and Social Change 155, 11999. https://doi.org/10.1016/j.techfore.2020.119995.

Foerster, O. (1934). Über die Bedeutung und Reichweite des Lokalisationsprinzips im Nervensystem. Verhandlungen der Deutschen Gesellschaft für Innere Medizin 46, 117-211.

Franceschini, A., Costantini, I., Pavone, F.S., and Silvestri, L. (2020). Dissecting Neuronal Activation on a Brain-Wide Scale With Immediate Early Genes. Front. Neurosci. 14. $10.3389 /$ fnins. 2020.569517.

Frégnac, Y., and Bathellier, B. (2015). Cortical Correlates of Low-Level Perception: From Neural Circuits to Percepts. Neuron 88, 110-126. 10.1016/j.neuron.2015.09.041.

Friston, K., Levin, M., Sengupta, B., and Pezzulo, G. (2015). Knowing one's place: a freeenergy approach to pattern regulation. J R Soc Interface 12. 10.1098/rsif.2014.1383.

Friston, K.J. (2011). Functional and effective connectivity: a review. Brain Connect 1, 1336. 10.1089/brain.2011.0008.

Friston, K.J., Harrison, L., and Penny, W. (2003). Dynamic causal modelling. Neuroimage 19, 1273-1302.

Friston, K.J., Parr, T., and de Vries, B. (2017). The graphical brain: Belief propagation and active inference. Netw Neurosci 1, 381-414. 10.1162/NETN_a_00018.

Furber, S., and Bogdan, P. (2020). SpiNNaker: A Spiking Neural Network Architecture (now publishers). http://dx.doi.org/10.1561/9781680836523.

Galadí, J.A., Silva Pereira, S., Sanz Perl, Y., Kringelbach, M.L., Gayte, I., Laufs, H., Tagliazucchi, E., Langa, J.A., and Deco, G. (2021). Capturing the non-stationarity of whole-brain dynamics underlying human brain states. Neuroimage 244, 118551. https://doi.org/10.1016/j.neuroimage.2021.118551.

Galison, P., and Hevly, B. (1992). The Growth of Large-Scale Research (Stanford University Press).

Gidon, A., Zolnik, T.A., Fidzinski, P., Bolduan, F., Papoutsi, A., Poirazi, P., Holtkamp, M., Vida, I., and Larkum, M.E. (2020). Dendritic action potentials and computation in human layer 2/3 cortical neurons. Science 367, 83-87. 10.1126/science.aax6239.

Goldman, J.S., Kusch, L., Yalçinkaya, B.H., Depannemaecker, D., Nghiem, T.-A.E., Jirsa, V., and Destexhe, A. (2021). A comprehensive neural simulation of slow-wave sleep and highly responsive wakefulness dynamics. bioRxiv, 2021.2008.2031.458365. 10.1101/2021.08.31.458365.

Goldman, J.S., Tort-Colet, N., di Volo, M., Susin, E., Bouté, J., Dali, M., Carlu, M., Nghiem, T.A., Górski, T., and Destexhe, A. (2019). Bridging Single Neuron Dynamics to Global Brain States. Front Syst Neurosci 13, 75. 10.3389/fnsys.2019.00075.

Goulas, A., Zilles, K., and Hilgetag, C.C. (2018). Cortical Gradients and Laminar Projections in Mammals. Trends Neurosci. 41, 775-788. 10.1016/j.tins.2018.06.003. 
1173

1174

1175

1176

1177

1178

1179

1180

1181

1182

1183

1184

1185

1186

1187

1188

1189

1190

1191

1192

1193

1194

1195

1196

1197

1198

1199

1200

1201

1202

1203

1204

1205

1206

1207

1208

1209

1210

1211

1212

1213

1214

1215

1216

1217

1218

1219

1220

1221

1222

1223

1224

1225

1226

1227

Grillner, S. (2014). Megascience efforts and the brain. Neuron 82, 1209-1211. 10.1016/j.neuron.2014.05.045.

Grillner, S., and Robertson, B. (2016). The Basal Ganglia Over 500 Million Years. Curr. Biol. 26, R1088-R1100. 10.1016/j.cub.2016.06.041.

Grossberg, S. (1988). Nonlinear neural networks: Principles, mechanisms, and architectures. Neural Networks 1, 17-61. https://doi.org/10.1016/0893-6080(88)90021-4.

Guevara, M., Román, C., Houenou, J., Duclap, D., Poupon, C., Mangin, J.F., and Guevara, P. (2017). Reproducibility of superficial white matter tracts using diffusion-weighted imaging tractography. Neuroimage 147, 703-725. 10.1016/j.neuroimage.2016.11.066.

Guevara, P., Duclap, D., Poupon, C., Marrakchi-Kacem, L., Fillard, P., Le Bihan, D., Leboyer, M., Houenou, J., and Mangin, J.F. (2012). Automatic fiber bundle segmentation in massive tractography datasets using a multi-subject bundle atlas. Neuroimage 61, 10831099. 10.1016/j.neuroimage.2012.02.071.

Haken, H. (1983). Synergetics: An introduction: Nonequilibrium phase transitions and selforganization in physics, chemistry, and biology 3rd rev. Edition (Springer).

Hansen, E.C.A., Battaglia, D., Spiegler, A., Deco, G., and Jirsa, V.K. (2015). Functional connectivity dynamics: Modeling the switching behavior of the resting state. Neuroimage 105, 525-535. https://doi.org/10.1016/j.neuroimage.2014.11.001.

Hashemi, M., Vattikonda, A.N., Sip, V., Diaz-Pier, S., Peyser, A., Wang, H., Guye, M., Bartolomei, F., Woodman, M.M., and Jirsa, V.K. (2021). On the influence of prior information evaluated by fully Bayesian criteria in a personalized whole-brain model of epilepsy spread. PLoS Comput Biol 17, e1009129. 10.1371/journal.pcbi.1009129.

Hashemi, M., Vattikonda, A.N., Sip, V., Guye, M., Bartolomei, F., Woodman, M.M., and Jirsa, V.K. (2020). The Bayesian Virtual Epileptic Patient: A probabilistic framework designed to infer the spatial map of epileptogenicity in a personalized large-scale brain model of epilepsy spread. Neuroimage 217, 116839. 10.1016/j.neuroimage.2020.116839.

Helias, M., Kunkel, S., Masumoto, G., Igarashi, J., Eppler, J.M., Ishii, S., Fukai, T., Morrison, A., and Diesmann, M. (2012). Supercomputers ready for use as discovery machines for neuroscience. Front Neuroinform 6, 26. 10.3389/fninf.2012.00026.

Hodge, R.D., Bakken, T.E., Miller, J.A., Smith, K.A., Barkan, E.R., Graybuck, L.T., Close, J.L., Long, B., Johansen, N., Penn, O., et al. (2019). Conserved cell types with divergent features in human versus mouse cortex. Nature 573, 61-68. 10.1038/s41586-019-1506-7.

Huys, R., Perdikis, D., and Jirsa, V.K. (2014). Functional architectures and structured flows on manifolds: a dynamical framework for motor behavior. Psychol. Rev. 121, 302-336. $10.1037 / \mathrm{a} 0037014$.

Jaramillo, J., Mejias, J.F., and Wang, X.J. (2019). Engagement of Pulvino-cortical Feedforward and Feedback Pathways in Cognitive Computations. Neuron 101, 321336.e329. 10.1016/j.neuron.2018.11.023.

Jirsa, V.K. (2008). Dispersion and time delay effects in synchronized spike-burst networks. Cogn. Neurodyn. 2, 29-38. 10.1007/s11571-007-9030-0.

Jirsa, V.K., Proix, T., Perdikis, D., Woodman, M.M., Wang, H., Gonzalez-Martinez, J., Bernard, C., Benar, C., Guye, M., Chauvel, P., and Bartolomei, F. (2017). The Virtual Epileptic Patient: Individualized whole-brain models of epilepsy spread. Neuroimage 145, 377-388. 10.1016/j.neuroimage.2016.04.049.

Kiebel, S., and Friston, K. (2011). Free Energy and Dendritic Self-Organization. Front. Syst. Neurosci. 5. 10.3389/fnsys.2011.00080.

Klein, B., Hoel, E., Swain, A., Griebenow, R., and Levin, M. (2021). Evolution and emergence: higher order information structure in protein interactomes across the tree of life. Integrative Biology. 10.1093/intbio/zyab020.

Kroner, A., Senden, M., Driessens, K., and Goebel, R. (2020). Contextual encoder-decoder network for visual saliency prediction. Neural Networks 129, 261-270. https://doi.org/10.1016/j.neunet.2020.05.004.

Larivière, S., Vos de Wael, R., Paquola, C., Hong, S.J., Mišić, B., Bernasconi, N., Bernasconi, A., Bonilha, L., and Bernhardt, B.C. (2019). Microstructure-Informed Connectomics: Enriching Large-Scale Descriptions of Healthy and Diseased Brains. Brain 
Connect 9, 113-127. 10.1089/brain.2018.0587

Li, A., Gong, H., Zhang, B., Wang, Q., Yan, C., Wu, J., Liu, Q., Zeng, S., and Luo, Q. (2010). Micro-optical sectioning tomography to obtain a high-resolution atlas of the mouse brain. Science 330, 1404-1408.

Lichtheim, L. (1885). On aphasia. Brain 7, 433-484.

Littlejohns, T.J., Holliday, J., Gibson, L.M., Garratt, S., Oesingmann, N., Alfaro-Almagro, F., Bell, J.D., Boultwood, C., Collins, R., Conroy, M.C., et al. (2020). The UK Biobank imaging enhancement of 100,000 participants: rationale, data collection, management and future directions. Nature communications 11, 2624. 10.1038/s41467-020-15948-9.

Llinás, R., Ribary, U., Contreras, D., and Pedroarena, C. (1998). The neuronal basis for consciousness. Philosophical transactions of the Royal Society of London. Series B, Biological sciences 353, 1841-1849. 10.1098/rstb.1998.0336.

Lombardo, D., Cassé-Perrot, C., Ranjeva, J.P., Le Troter, A., Guye, M., Wirsich, J., Payoux, P., Bartrés-Faz, D., Bordet, R., Richardson, J.C., et al. (2020). Modular slowing of resting-state dynamic functional connectivity as a marker of cognitive dysfunction induced by sleep deprivation. Neuroimage 222, 117155. 10.1016/j.neuroimage.2020.117155.

Mainen, Z.F., and Pouget, A. (2014). Put brain project back on course. Nature 511, 534534. $10.1038 / 511534 b$.

Markram, H., Meier, K., Lippert, T., Grillner, S., Frackowiak, R., Dehaene, S., Knoll, A., Sompolinsky, H., Verstreken, K., DeFelipe, J., et al. (2011). Introducing the Human Brain Project. Procedia Comput Sci 7, 39-42. 10.1016/j.procs.2011.12.015.

Markram, H., Muller, E., Ramaswamy, S., Reimann, M.W., Abdellah, M., Sanchez, C.A., Ailamaki, A., Alonso-Nanclares, L., Antille, N., Arsever, S., et al. (2015). Reconstruction and Simulation of Neocortical Microcircuitry. Cell 163, 456-492. 10.1016/j.cell.2015.09.029.

Markram, H., and Perin, R. (2011). Innate neural assemblies for lego memory. Frontiers in neural circuits 5, 6. 10.3389/fncir.2011.00006.

Menzel, M., Axer, M., Amunts, K., De Raedt, H., and Michielsen, K. (2019). Diattenuation Imaging reveals different brain tissue properties. Sci. Rep. 9, 1939. 10.1038/s41598-01938506-w.

Muratore, P., Capone, C., and Paolucci, P.S. (2021). Target spike patterns enable efficient and biologically plausible learning for complex temporal tasks. PLoS One 16, e0247014. 10.1371 /journal.pone.0247014.

Okano, H., Miyawaki, A., and Kasai, K. (2015). Brain/MINDS: brain-mapping project in Japan. Philosophical Transactions of the Royal Society B: Biological Sciences 370, 20140310. doi:10.1098/rstb.2014.0310.

Olmi, S., Petkoski, S., Guye, M., Bartolomei, F., and Jirsa, V. (2019). Controlling seizure propagation in large-scale brain networks. PLoS Comput. Biol. 15, e1006805. 10.1371/journal.pcbi.1006805.

Palomero-Gallagher, N., and Zilles, K. (2019). Cortical layers: Cyto-, myelo-, receptor- and synaptic architecture in human cortical areas. Neuroimage 197, 716-741. https://doi.org/10.1016/j.neuroimage.2017.08.035.

Pearson, M.J., Dora, S., Struckmeier, O., Knowles, T.C., Mitchinson, B., Tiwari, K., Kyrki, V., Bohte, S., and Pennartz, C.M.A. (2021). Multimodal Representation Learning for Place Recognition Using Deep Hebbian Predictive Coding. Front Robot Al 8, 732023. $10.3389 /$ frobt.2021.732023.

Pennartz, C.M. (2015). The brain's representational power - on consciousness and the integration of modalities (MIT Press).

Pennartz, C.M.A., Dora, S., Muckli, L., and Lorteije, J.A.M. (2019a). Towards a Unified View on Pathways and Functions of Neural Recurrent Processing. Trends Neurosci. 42, 589603. 10.1016/j.tins.2019.07.005.

Pennartz, C.M.A., Farisco, M., and Evers, K. (2019b). Indicators and Criteria of Consciousness in Animals and Intelligent Machines: An Inside-Out Approach. Front. Syst. Neurosci. 13. 10.3389/fnsys.2019.00025.

Petkoski, S., and Jirsa, V. (2021). Normalising the brain connectome for communication 
1283

1284

1285

1286

1287

1288

1289

1290

1291

1292

1293

1294

1295

1296

1297

1298

1299

1300

1301

1302

1303

1304

1305

1306

1307

1308

1309

1310

1311

1312

1313

1314

1315

1316

1317

1318

1319

1320

1321

1322

1323

1324

1325

1326

1327

1328

1329

1330

1331

1332

1333

1334

1335

1336

1337

through synchronization. . Network Neuroscience in press.

Petkoski, S., and Jirsa, V.K. (2019). Transmission time delays organize the brain network synchronization. Philosophical Transactions of the Royal Society A: Mathematical, Physical and Engineering Sciences 377, 20180132. doi:10.1098/rsta.2018.0132.

Pillai, A.S., and Jirsa, V.K. (2017). Symmetry Breaking in Space-Time Hierarchies Shapes Brain Dynamics and Behavior. Neuron 94, 1010-1026. 10.1016/j.neuron.2017.05.013.

Pinho, A., Amadon, A., Ruest, T., Fabre, M., Dohmatob, E., Denghien, I., Ginisty, C., Becuwe-Desmidt, S., Roger, S., Laurier, L., et al. (2020). Individual Brain Charting (IBC, release 2) EBRAINS, 10.25493/XX28-VJ1.

Pinho, A.L., Amadon, A., Fabre, M., Dohmatob, E., Denghien, I., Torre, J.J., Ginisty, C., Becuwe-Desmidt, S., Roger, S., Laurier, L., et al. (2021a). Subject-specific segregation of functional territories based on deep phenotyping. Hum. Brain Mapp. 42, 841-870. 10.1002/hbm.25189.

Pinho, A.L., Amadon, A., Ruest, T., Fabre, M., Dohmatob, E., Denghien, I., Ginisty, C., Becuwe-Desmidt, S., Roger, S., Laurier, L., et al. (2018). Individual Brain Charting, a highresolution fMRI dataset for cognitive mapping. Scientific Data 5, 180105. 10.1038/sdata.2018.105.

Pinho, A.L., Shankar, S., Richard, H., Amadon, A., Nishimoto, S., Huth, A.G., Eickenberg, M., Denghien, I., Torre, J.J., Aggarwal, H., et al. (2021b). Individual Brain Charting (IBC, release 3) EBRAINS, 10.25493/SM37-TS4.

Potjans, T.C., and Diesmann, M. (2014). The cell-type specific cortical microcircuit: relating structure and activity in a full-scale spiking network model. Cereb. Cortex 24, 785806. $10.1093 /$ cercor/bhs358.

Proix, T., Bartolomei, F., Guye, M., and Jirsa, V.K. (2017). Individual brain structure and modelling predict seizure propagation. Brain 140, 641-654. 10.1093/brain/awx004.

Quaglio, G., Toia, P., Moser, E.I., Karapiperis, T., Amunts, K., Okabe, S., Poo, M.-m., Rah, J.-C., Koninck, Y.D., Ngai, J., et al. (2021). The International Brain Initiative: enabling collaborative science. The Lancet Neurology 20, 985-986. 10.1016/S1474-4422(21)00389-6.

Ramsey, R., and Ward, R. (2020). Challenges and opportunities for top-down modulation research in cognitive psychology. Acta Psychol. (Amst.) 209, 103118. 10.1016/j.actpsy.2020.103118.

Redolfi, A., De Francesco, S., Palesi, F., Galluzzi, S., Muscio, C., Castellazzi, G., Tiraboschi, P., Savini, G., Nigri, A., Bottini, G., et al. (2020). Medical Informatics Platform (MIP): A Pilot Study Across Clinical Italian Cohorts. Front. Neurol. 11, 1021. 10.3389/fneur.2020.01021.

Rhodes, O., Peres, L., Rowley, A.G.D., Gait, A., Plana, L.A., Brenninkmeijer, C., and Furber, S.B. (2020). Real-time cortical simulation on neuromorphic hardware. Philosophical Transactions of the Royal Society A: Mathematical, Physical and Engineering Sciences 378, 20190160. doi:10.1098/rsta.2019.0160.

Rockland, K.S. (2020). What we can learn from the complex architecture of single axons. Brain Structure and Function 225, 1327-1347. 10.1007/s00429-019-02023-3.

Rockland, K.S., and DeFelipe, J. (2018). Editorial: Why Have Cortical Layers? What Is the Function of Layering? Do Neurons in Cortex Integrate Information Across Different Layers? Frontiers in Neuroanatomy 12. 10.3389/fnana.2018.00078.

Rossetti, G., Kless, A., Lai, L., Outeiro, T.F., and Carloni, P. (2019). Investigating targets for neuropharmacological intervention by molecular dynamics simulations. Biochem. Soc. Trans. 47, 909-918. 10.1042/bst20190048.

Salles, A., Bjaalie, J.G., Evers, K., Farisco, M., Fothergill, B.T., Guerrero, M., Maslen, H., Muller, J., Prescott, T., Stahl, B.C., et al. (2019a). The Human Brain Project: Responsible brain research for the benefit of society. Neuron 101, 380-384. https://doi.org/10.1016/j.neuron.2019.01.005.

Salles, A., Evers, K., and Farisco, M. (2019b). The Need for a Conceptual Expansion of Neuroethics. AJOB Neurosci. 10, 126-128. 10.1080/21507740.2019.1632972.

Sanchez-Vives, M.V., Massimini, M., and Mattia, M. (2017). Shaping the default activity pattern of the cortical network. Neuron 94, 993-1001. 
https: / /doi.org/10.1016/j.neuron.2017.05.015.

Sanz-Leon, P., Knock, S.A., Spiegler, A., and Jirsa, V.K. (2015). Mathematical framework for large-scale brain network modeling in The Virtual Brain. Neuroimage 111, 385-430. 10.1016/j.neuroimage.2015.01.002.

Schiffer, C., Spitzer, H., Kiwitz, K., Unger, N., Wagstyl, K., Evans, A.C., Harmeling, S., Amunts, K., and Dickscheid, T. (2021). Convolutional neural networks for cytoarchitectonic brain mapping at large scale. Neuroimage 240, 118327. https://doi.org/10.1016/j.neuroimage.2021.118327.

Schneider, M., Kemper, V.G., Emmerling, T.C., De Martino, F., and Goebel, R. (2019). Columnar clusters in the human motion complex reflect consciously perceived motion axis. Proc. Natl. Acad. Sci. U. S. A. 116, 5096-5101. 10.1073/pnas.1814504116.

Sip, V., Hashemi, M., Vattikonda, A.N., Woodman, M.M., Wang, H., Scholly, J., Medina Villalon, S., Guye, M., Bartolomei, F., and Jirsa, V.K. (2021). Data-driven method to infer the seizure propagation patterns in an epileptic brain from intracranial electroencephalography. PLoS Comput Biol 17, e1008689. 10.1371/journal.pcbi.1008689.

Spiegler, A., Hansen, E.C.A., Bernard, C., McIntosh, A.R., and Jirsa, V.K. (2016). Selective Activation of Resting-State Networks following Focal Stimulation in a Connectome-Based Network Model of the Human Brain. eneuro 3, ENEURO.0068-0016.2016. 10.1523/eneuro.0068-16.2016.

Sporns, O., Tononi, G., and Kötter, R. (2005). The human connectome: A structural description of the human brain. PLoS Comput Biol 1, e42. 10.1371/journal.pcbi.0010042. Stahl, B. (2021). Artificial Intelligence for a Better Future. An Ecosystem Perspective on the Ethics of Al and Emerging Digital Technologies (Springer).

Stefanovski, L., Meier, J.M., Pai, R.K., Triebkorn, P., Lett, T., Martin, L., Bülau, K., Hofmann-Apitius, M., Solodkin, A., McIntosh, A.R., and Ritter, P. (2021). Bridging Scales in Alzheimer's Disease: Biological Framework for Brain Simulation With The Virtual Brain. Front Neuroinform 15, 630172. 10.3389/fninf.2021.630172.

Storm, J.F., Boly, M., Casali, A.G., Massimini, M., Olcese, U., Pennartz, C.M.A., and Wilke, M. (2017). Consciousness Regained: Disentangling Mechanisms, Brain Systems, and Behavioral Responses. J. Neurosci. 37, 10882-10893. 10.1523/Jneurosci.1838-17.2017.

Sun, Z.Y., Pinel, P., Riviere, D., Moreno, A., Dehaene, S., and Mangin, J.F. (2016). Linking morphological and functional variability in hand movement and silent reading. Brain Struct Funct 221, 3361-3371. 10.1007/s00429-015-1106-8.

Teeters, J.L., Godfrey, K., Young, R., Dang, C., Friedsam, C., Wark, B., Asari, H., Peron, S., Li, N., Peyrache, A., et al. (2015). Neurodata Without Borders: Creating a Common Data Format for Neurophysiology. Neuron 88, 629-634. 10.1016/j.neuron.2015.10.025.

Thompson, P.M., Jahanshad, N., Ching, C.R.K., Salminen, L.E., Thomopoulos, S.I., Bright, J., Baune, B.T., Bertolín, S., Bralten, J., Bruin, W.B., et al. (2020). ENIGMA and global neuroscience: A decade of large-scale studies of the brain in health and disease across more than 40 countries. Transl Psychiatry 10, 100. 10.1038/s41398-020-0705-1.

Tononi, G., and Koch, C. (2015). Consciousness: here, there and everywhere? Philosophical Transactions of the Royal Society of London B: Biological Sciences 370. 10.1098/rstb.2014.0167.

van Albada, S.J., Rowley, A.G., Senk, J., Hopkins, M., Schmidt, M., Stokes, A.B., Lester, D.R., Diesmann, M., and Furber, S.B. (2018). Performance comparison of the digital neuromorphic hardware SpiNNaker and the neural network simulation software NEST for a full-scale cortical microcircuit model. Front. Neurosci. 12. ARTN 291 10.3389/fnins.2018.00291.

Van Essen, D.C., Smith, S.M., Barch, D.M., Behrens, T.E., Yacoub, E., and Ugurbil, K. (2013). The WU-Minn Human Connectome Project: an overview. Neuroimage 80, 62-79. 10.1016/j.neuroimage.2013.05.041.

Vattikonda, A.N., Hashemi, M., Sip, V., Woodman, M.M., Bartolomei, F., and Jirsa, V.K. (2021). Identifying spatio-temporal seizure propagation patterns in epilepsy using Bayesian inference. Commun Biol 4, 1244. 10.1038/s42003-021-02751-5.

Vogelstein, J.T., Mensh, B., Hausser, M., Spruston, N., Evans, A., Kording, K., Amunts, K., 
1393

1394

1395

1396

1397

1398

1399

1400

1401

1402

1403

1404

1405

1406

1407

1408

1409

1410

1411

1412

1413

1414

1415

1416

1417

1418

1419

1420

1421

1422

1423

1424

1425

Ebell, C., Muller, J., Telefont, M., et al. (2016). To the Cloud! A grassroots proposal to accelerate brain science discovery. Neuron 92, 622-627. 10.1016/j.neuron.2016.10.033. Vogt, C., and Vogt, O. (1926). Die vergleichend-architektonische und die vergleichendreizphysiologische Felderung der Großhirnrinde unter besonderer Beraücksichtigung der menschlichen. Die Naturwissenschaften 14, 1192-1195.

von der Malsburg, C. (1999). The What and Why of Binding: The Modeler's Perspective. Neuron 24, 95-104. 10.1016/S0896-6273(00)80825-9.

Wagstyl, K., Larocque, S., Cucurull, G., Lepage, C., Cohen, J.P., Bludau, S., PalomeroGallagher, N., Lewis, L.B., Funck, T., Spitzer, H., et al. (2020). BigBrain 3D atlas of cortical layers: Cortical and laminar thickness gradients diverge in sensory and motor cortices. PLoS Biol 18, e3000678. 10.1371/journal.pbio.3000678.

Wernicke, C. (1874). Der aphasische Symptomencomplex. Eine psychologische Studie auf anatomischer Basis (Springer Verlag).

Wybo, W.A., Jordan, J., Ellenberger, B., Marti Mengual, U., Nevian, T., and Senn, W. (2021). Data-driven reduction of dendritic morphologies with preserved dendro-somatic responses. Elife 10. 10.7554/eLife.60936.

Yuste, R., Hawrylycz, M., Aalling, N., Aguilar-Valles, A., Arendt, D., Armañanzas, R., Ascoli, G.A., Bielza, C., Bokharaie, V., Bergmann, T.B., et al. (2020). A community-based transcriptomics classification and nomenclature of neocortical cell types. Nature Neuroscience 23, 1456-1468. 10.1038/s41593-020-0685-8.

Zenke, F., Bohté, S.M., Clopath, C., Comșa, I.M., Göltz, J., Maass, W., Masquelier, T., Naud, R., Neftci, E.O., Petrovici, M.A., et al. (2021). Visualizing a joint future of neuroscience and neuromorphic engineering. Neuron 109, 571-575. 10.1016/j.neuron.2021.01.009.

Zhao, L., Batta, I., Matloff, W., O'Driscoll, C., Hobel, S., and Toga, A.W. (2020). Neuroimaging PheWAS (Phenome-Wide Association Study): A Free Cloud-Computing Platform for Big-Data, Brain-Wide Imaging Association Studies. Neuroinformatics. 10.1007/s12021-020-09486-4.

Zilles, K., and Amunts, K. (2009). Receptor mapping: Architecture of the human cerebral cortex. Curr. Opin. Neurol. 22, 331-339.

Zilles, K., and Amunts, K. (2013). Individual variability is not noise. TINS 17, 153-155. 10.1016/j.tics.2013.02.003. 


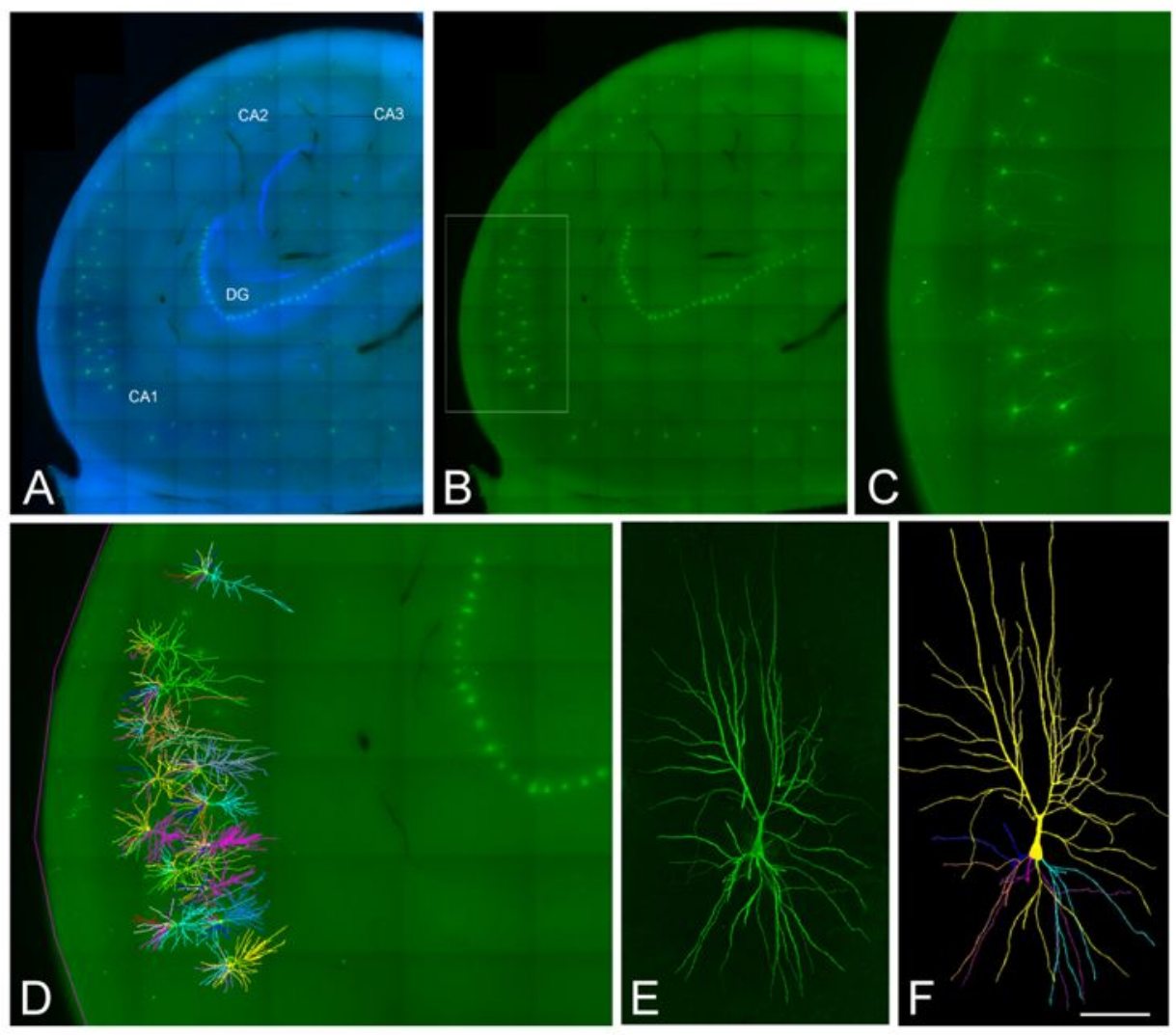




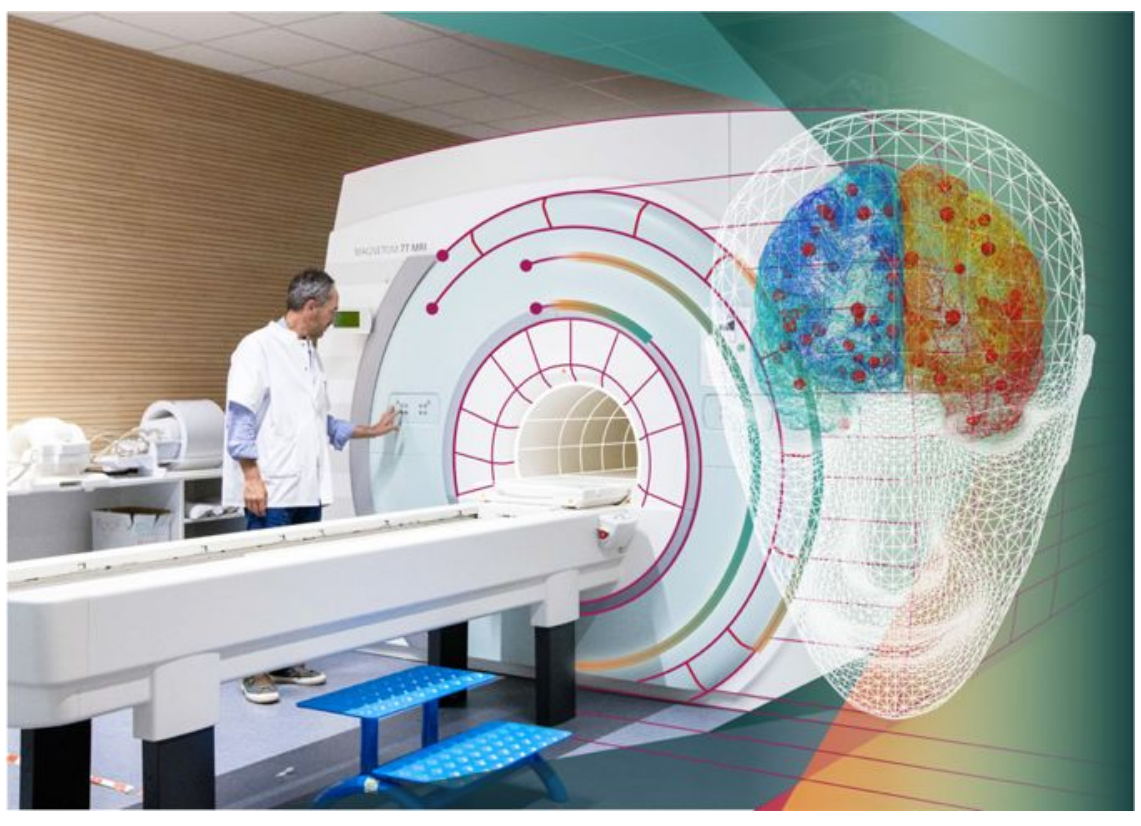




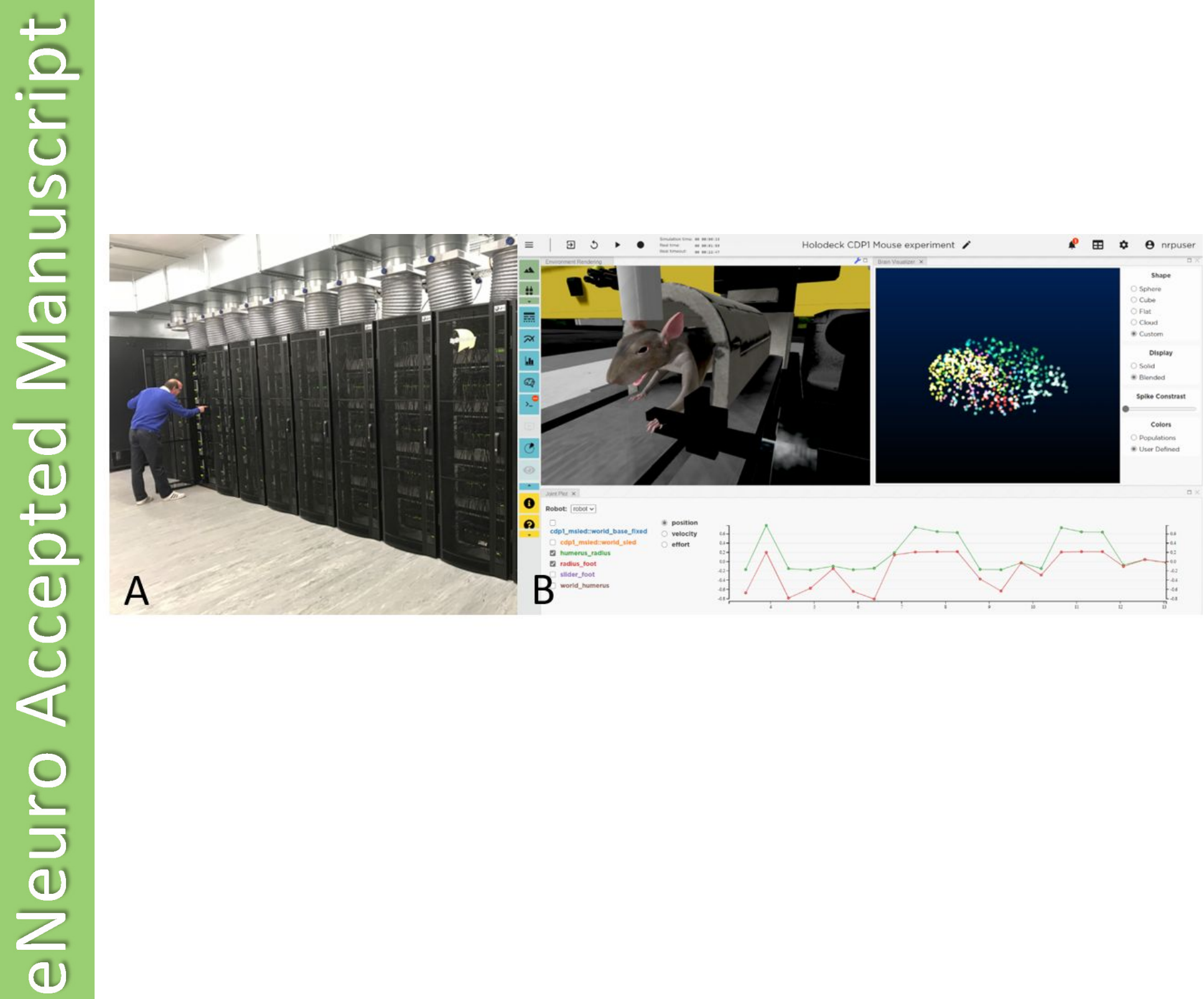




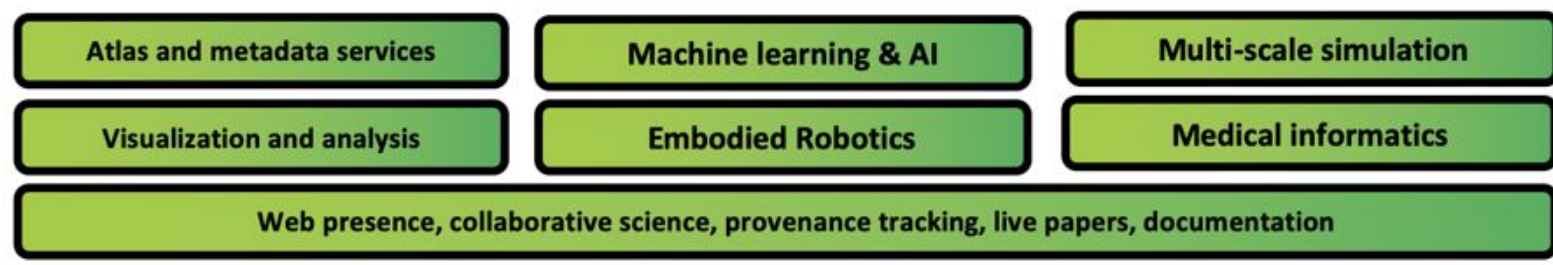

VMs + containers, source control, continues integration

\begin{tabular}{|c|c|}
\hline $\begin{array}{l}\text { neuromorphic computing: } \\
\text { BrainScales \& Spinnaker }\end{array}$ & $\begin{array}{l}\text { Interactive supercomputing } \\
\text { In }\end{array}$ \\
\hline
\end{tabular}

Massive HPC \& big data:

Tier 0 and 1 HPC resources 

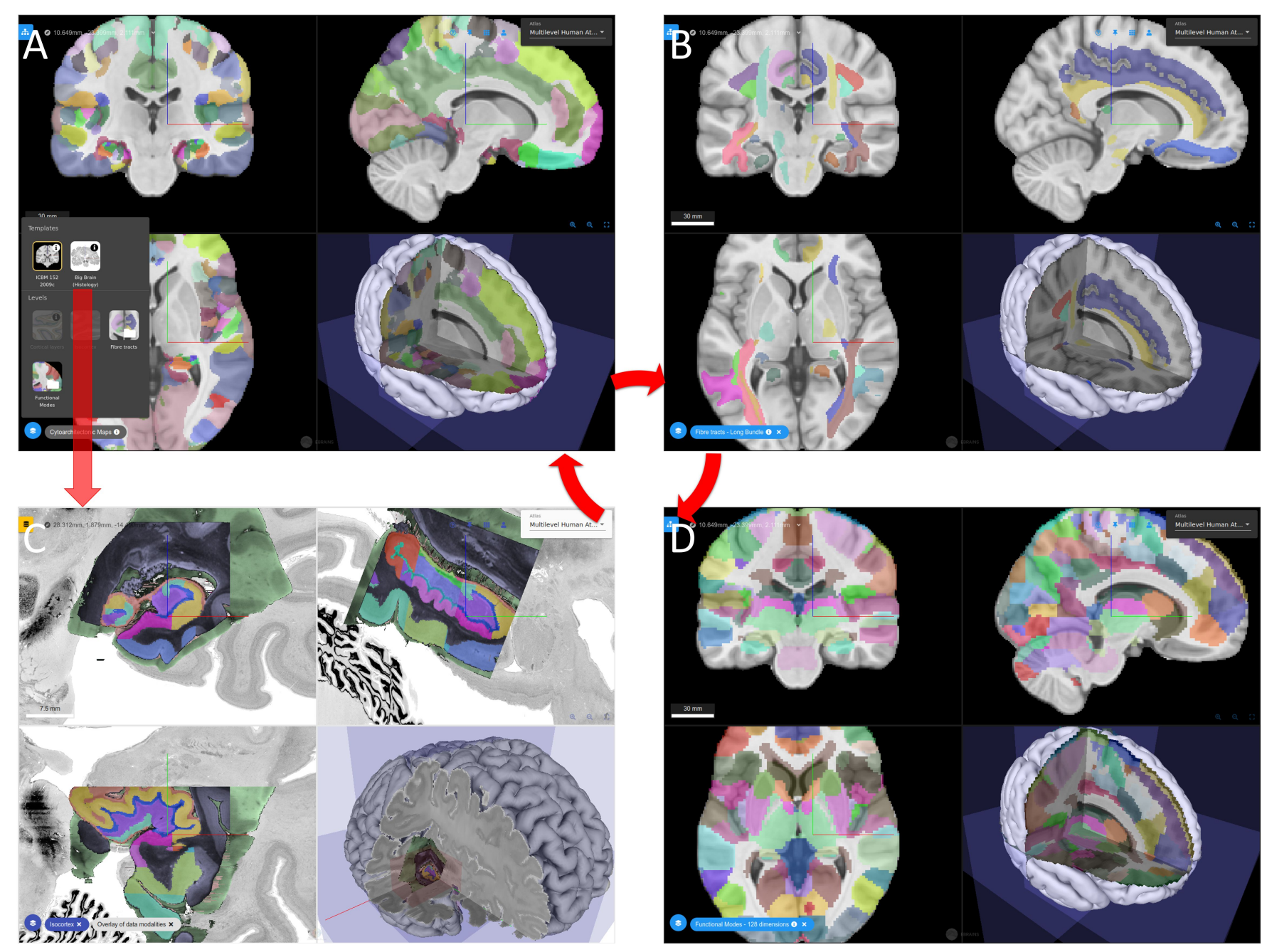
$e^{3}(4)$.

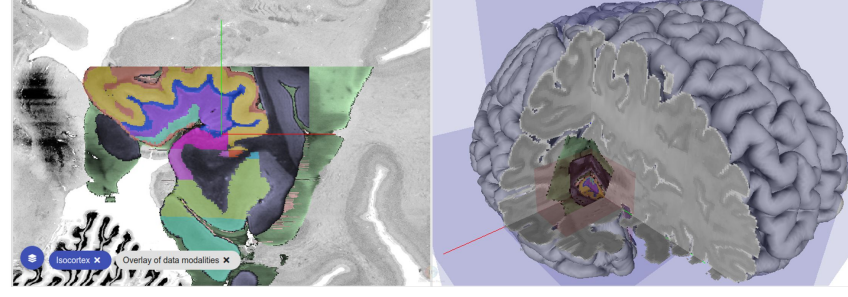

